\title{
TOPOLOGY THROUGH THE CENTURIES: LOW DIMENSIONAL MANIFOLDS
}

\author{
JOHN MILNOR \\ Based on the Abel Lecture at the \\ 2014 International Congress of Mathematicians in Seoul
}

\begin{abstract}
This note will provide a lightning tour through the centuries, concentrating on the study of manifolds of dimension 2, 3, and 4. Further comments and more technical details about many of the sections may be found in the Appendix.
\end{abstract}

\section{Part 1. Prelude to Topology}

The subject known as topology took shape in the 19th century, made dramatic progress during the 20th century, and is flourishing in the 21st. But before there was any idea of topology, there were isolated results which hinted that there should be such a field of study.

\subsection{Leonhard Euler, St. Petersburg, 1736.}

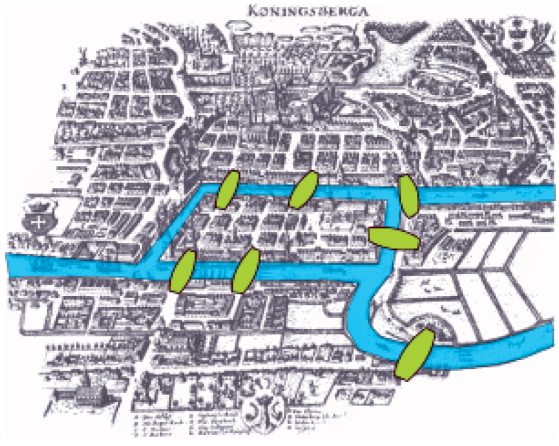

Königsberg in the 18th century

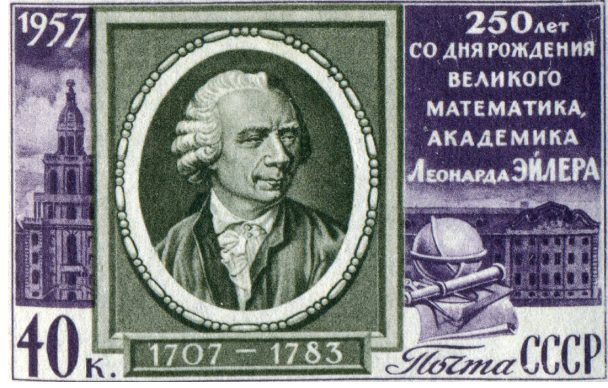

Euler

Perhaps the first topological statement in the mathematical literature came with Euler's solution to the problem of the Seven Bridges of Königsberg: the problem of taking a walk which traverses each of the seven bridges exactly once. In fact, Euler showed that no such walk is possible. The problem can be represented by

2010 Mathematics Subject Classification. Primary 57N05-57N13, Secondary 01A55, 01A60. 
a graph, as shown below, where each land mass is represented by a dot, and each bridge by an edge.

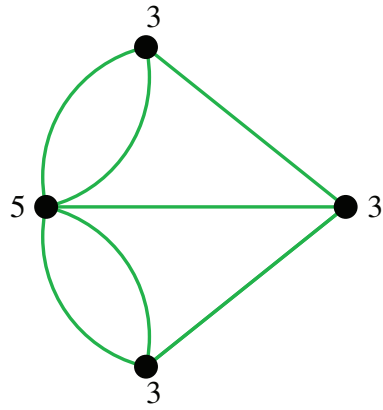

Graph for the bridge problem.
Theorem. There exists a path traversing each edge of such a graph exactly once if and only if the graph has at most two vertices which are "odd", in the sense that an odd number of edges meet there.

For the given graph, all four vertices are "odd", so no such path is possible. This theorem provides an excellent exercise for young mathematicians, since the proof is quite elementary, but not immediately obvious.

\subsection{Leonhard Euler, Berlin, 1752.}

Some years later, Euler described an equality which has played a fundamental role in topology.

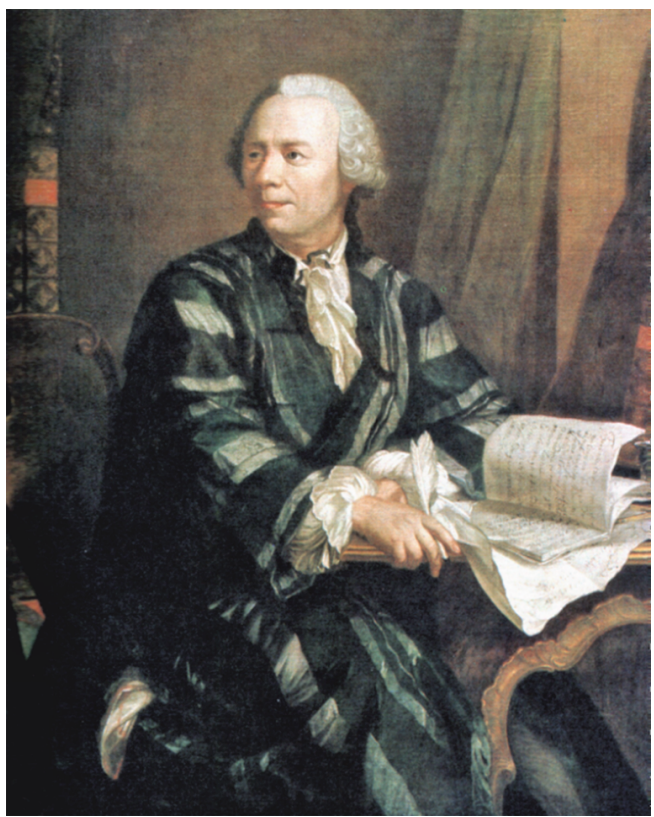

Theorem. For any convex polyhedron, the numbers of vertices, edges and faces are related by the equation

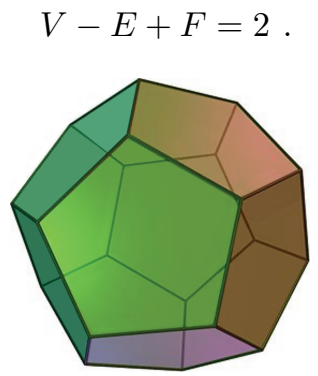

Example. The dodecahedron has 20 vertices, 30 edges, and 12 faces, with

$$
20-30+12=2 \text {. }
$$

Euler was far ahead of his time. More than a hundred years later, the Euler characteristic of an arbitrary finite cell complex $\mathbf{K}$ was defined as the integer

$$
\chi(\mathbf{K})=\#(\text { even dimensional cells })-\#(\text { odd dimensional cells }) .
$$


Only in the early twentieth century was it possible to prove that this Euler characteristic is a topological invariant. Two fundamental properties which are very easy to check from the definition are the following, where the first assumes that the $\mathbf{K}_{j}$ are subcomplexes of their union:

$$
\begin{aligned}
& \chi\left(\mathbf{K}_{1} \cup \mathbf{K}_{2}\right)=\chi\left(\mathbf{K}_{1}\right)+\chi\left(\mathbf{K}_{2}\right)-\chi\left(\mathbf{K}_{1} \cap \mathbf{K}_{2}\right) \quad \text { and } \\
& \chi\left(\mathbf{K}_{1} \times \mathbf{K}_{2}\right)=\chi\left(\mathbf{K}_{1}\right) \chi\left(\mathbf{K}_{2}\right) .
\end{aligned}
$$

As easy exercises, using the definition and the product property, one can show that the Euler characteristic of the $n$-dimensional cube $[0,1]^{n}$ is +1 , while the Euler characteristic of the boundary of this cube, which is topologically an $(n-1)$ dimensional sphere, is $1+(-1)^{n-1}$.

\subsection{Augustin Cauchy, École Polytechnique, Paris, 1825.}
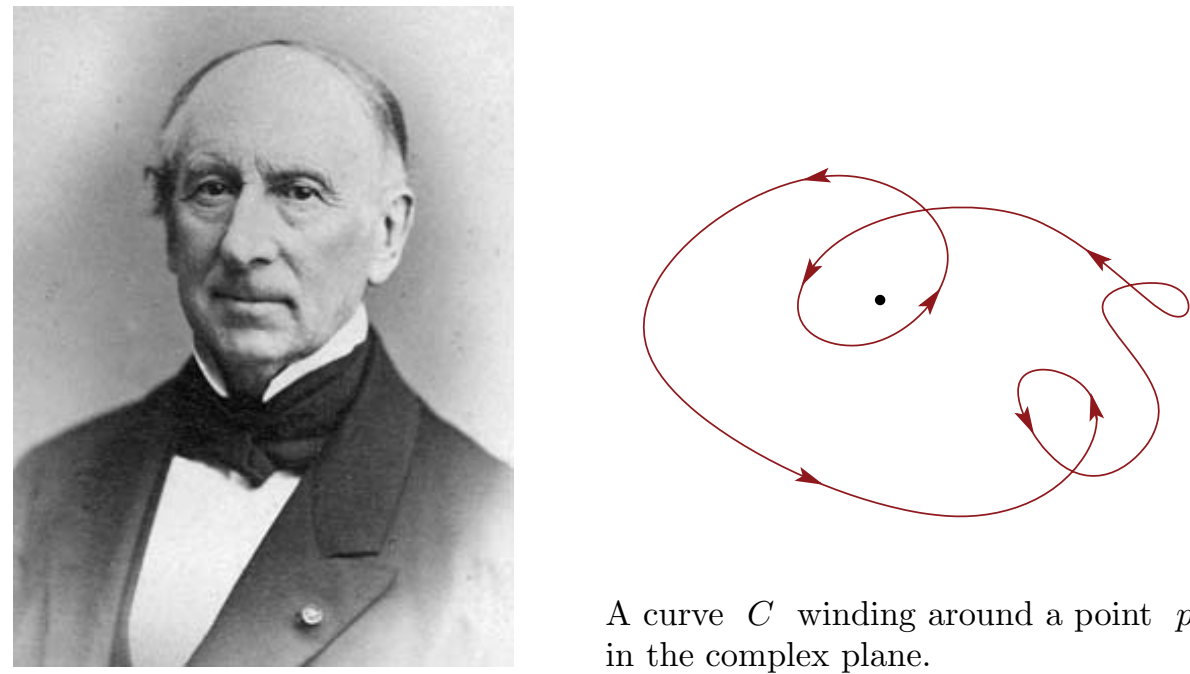

A curve $C$ winding around a point $p$ in the complex plane.

Cauchy was the first to give a precise definition of continuity, which is surely one of the most fundamental concepts of topology.

Furthermore, he described a topological invariant, the winding number of a loop $C$ around a point $p$, and computed it as the integral of a holomorphic differential form along $C$ :

$$
W_{C}(p)=\frac{1}{2 \pi i} \oint_{C} \frac{d z}{z-p} \in \mathbb{Z}
$$




\subsection{Carl Friedrich Gauss, Göttingen, 1833.}
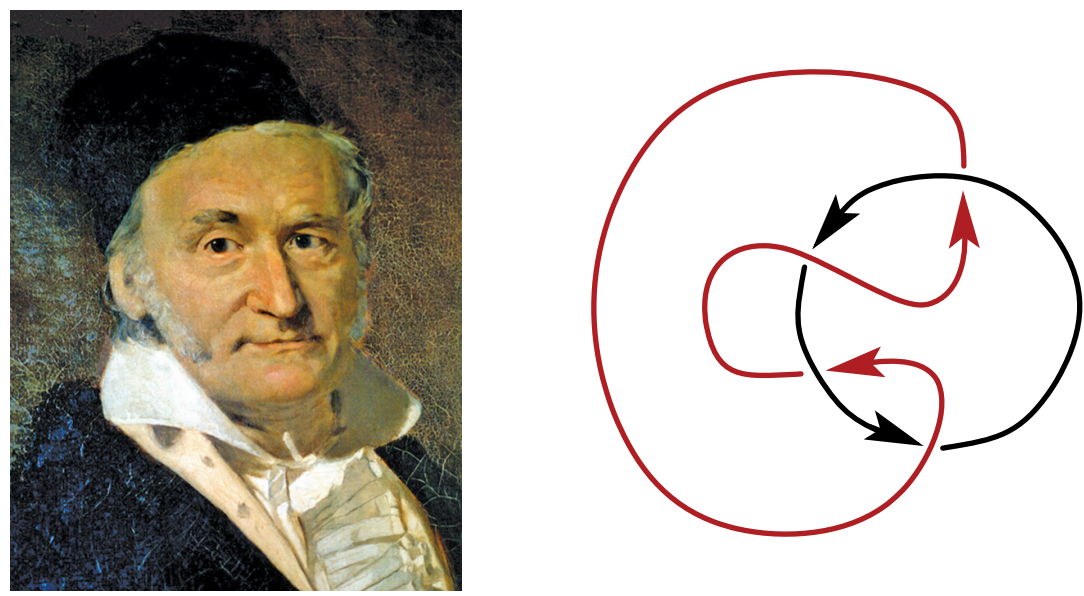

Gauss described a much more sophisticated topological invariant: the linking number $L$ between two disjoint oriented closed curves in $\mathbb{R}^{3}$. Inspired by his study of electromagnetism, he computed this number as a double integral

$$
L=\frac{1}{4 \pi} \iint_{x, y} \frac{(x-y) \cdot(d x \times d y)}{\|x-y\|^{3}} \in \mathbb{Z}
$$

Here $x$ varies around one of the curves and $y$ varies around the other.

\section{Part 2. Two-Dimensional Manifolds}

Only in the nineteenth century did it become clear that there should be studies of geometry, not only locally, but also in the large.

\subsection{Simon L'Huilier, Académie Impériale de Genève, 1812-1813.}

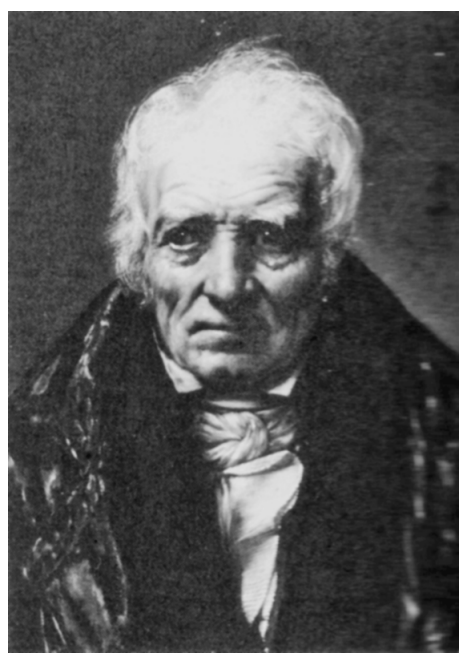

L'Huilier (also spelled Lhuilier) was probably the first person to consider the Euler characteristic for more general (non-convex) polyhedra. For the surface of a polyhedron in Euclidean 3-space which is "drilled through" by $n$ openings, he computed the Euler characteristic as $\chi=2-2 n$. In modern language, the boundary of such a polyhedron is a surface of genus $n$.

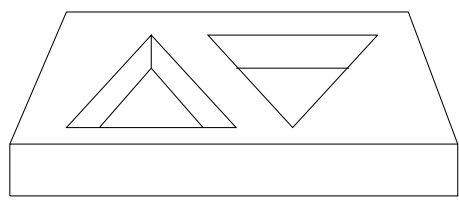




\subsection{Niels Henrik Abel, Norway, 1820s.}

The necessity of a global theory of surfaces became clear with the work of Abel, who studied the integrals of algebraic functions, for example of the form

$$
\int \frac{d x}{\sqrt{\left(x-a_{1}\right) \cdots\left(x-a_{n}\right)}}
$$

where the $a_{j}$ are distinct real or complex numbers. Today we would describe this as the integral $\int d x / y$ along a path in the smooth affine variety $V \subset \mathbb{C}^{2}$ defined by

$$
y^{2}=f(x)=\left(x-a_{1}\right) \cdots\left(x-a_{n}\right) .
$$

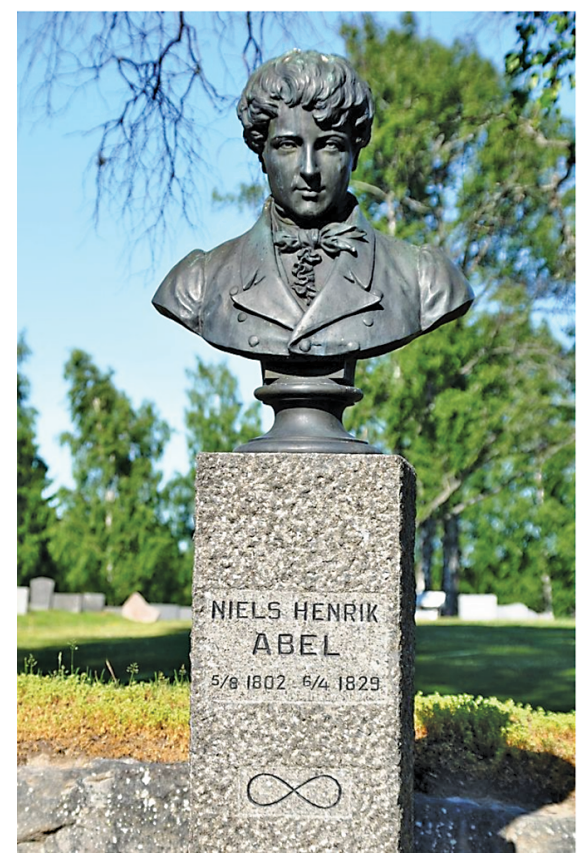

Abel memorial in Gjerstad
The integrand doesn't seem to make sense when $y=0$; but since

$$
2 y d y=f^{\prime}(x) d x,
$$

we can also write this integrand as $2 d y / f^{\prime}(x)$, which is perfectly well behaved when $y=0$. Nowadays we describe this expression

$$
\boldsymbol{\alpha}=\frac{d x}{y}=\frac{2 d y}{f^{\prime}(x)}
$$

as a holomorphic 1-form or Abelian differential on the variety $V$.

Given any such Abelian differential $\boldsymbol{\alpha}$ and any closed loop $L$ on $V$, we can integrate, so as to obtain a homomorphism

$$
L \mapsto \int_{L} \boldsymbol{\alpha}
$$

from the fundamental group $\pi_{1}(V)$ to the complex numbers.

However, much of this terminology was not available at the time. Abel died very early, and it was left to others to work out a suitable language for describing such constructions.

\subsection{Bernhard Riemann, Göttingen, 1857.}

The first person to take up the challenge was Riemann. In his 1851 dissertation, and in his 1857 paper on Abelian functions, he developed the concept of what we now call a Riemann surface. 


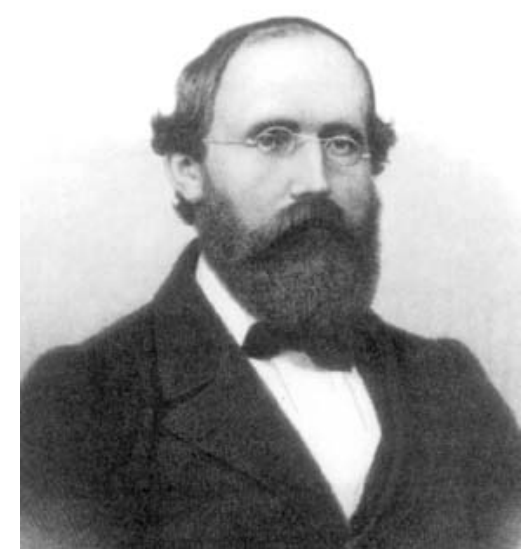

Below are copies of Riemann's illustrations for three bounded regions of the complex plane. He called a region simply connected if any cross-cut (that is any path through the region from one boundary point to another) necessarily separates the region. Similarly it is doubly connected if two cross-cuts are needed in order to necessarily separate the region, and so on.

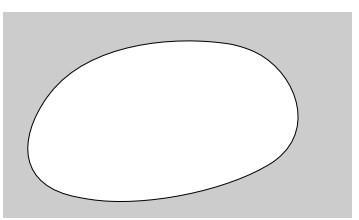

simply connected

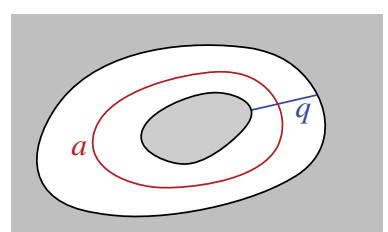

doubly connected

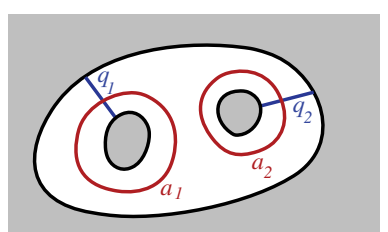

triply connected

More generally, he studied "Riemann surfaces" which lie over the plane:

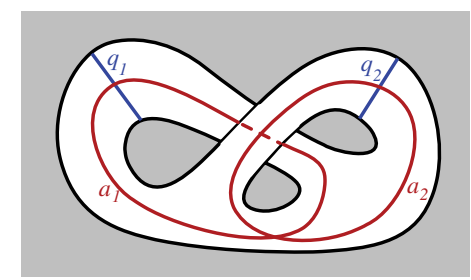

another triply connected surface

Riemann also considered the case of a closed surface $\mathcal{F}$. He described a procedure for cutting $\mathcal{F}$ open along a number of simple closed curves, which intersect at just one point, so that the cut-open surface is connected and simply connected. 

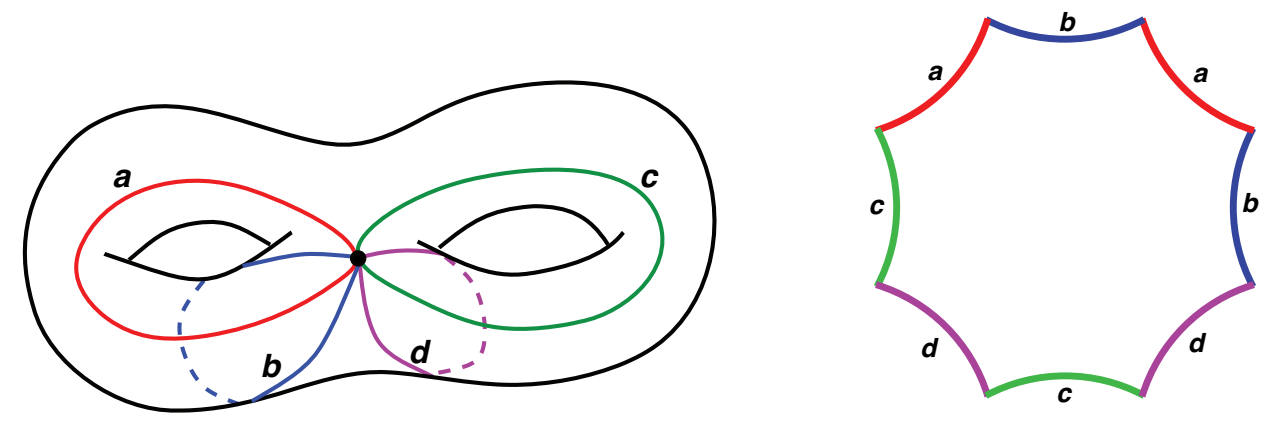

The number of such curves is always an even number $2 p$. In modern terminology, this describes the structure of a CW-cell complex 1 with one vertex, $2 p$ edges, and one 2-cell, yielding an Euler characteristic of $\chi=2-2 p$. This Riemann integer $p \geq 0$ is an invariant now known as the genus of $\mathcal{F}$, while $2 p$ is known as the one-dimensional Betti number.

\subsection{August Ferdinand Möbius, Leipzig, 1863.}

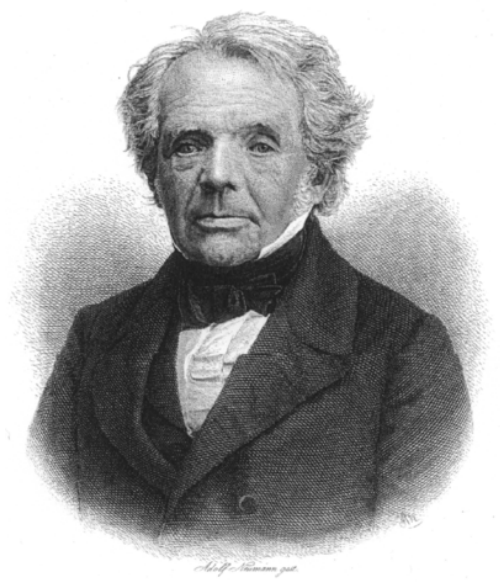

Although Riemann's pioneering ideas have influenced all subsequent work in this area, his writing gave few details and is not always easy to follow. A few years later, Möbius gave a presentation which seems much clearer to me, showing that smooth closed surfaces in 3-space can be classified by a clearly defined integer invariant.

Define the connectivity class of a closed surface $\mathcal{F} \subset \mathbb{R}^{3}$ as the smallest number $n$ such that any $n$ disjoint loops in $\mathcal{F}$ necessarily disconnect it. (In other words, we can choose $p=n-1$ closed loops which do not disconnect the surface; but any further disjoint loop will necessarily disconnect it. Here $p$ is again Riemann's invariant, the genus.)

Example. For the torus we have $p=1$, since we can cut one loop out of the torus without disconnecting it, but two disjoint loops will necessarily disconnect.

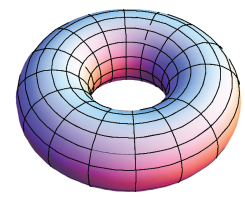

Theorem. Any two closed surfaces of the same connectivity class are "elementarily related".

\footnotetext{
${ }^{1}$ Compare Whitehead 1949b.
} 
The Möbius concept of "elementarily related", as defined below, is very awkward. In modern terms, what he surely wants to describe is the concept of $C^{1}$-diffeomorphism.

Definition. Two geometric figures are "elementarily related" if to any infinitely small element of any dimension in one figure there corresponds an infinitely small element in the other figure, such that two neighboring elements in one figure correspond to two elements in the other which also come together, ...

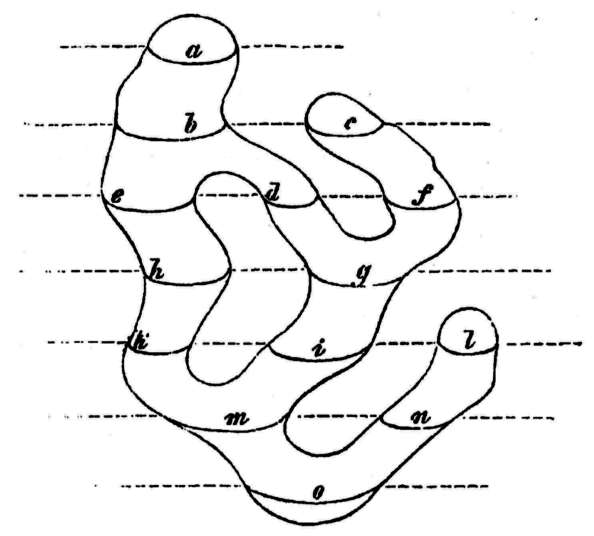

But the Möbius proof is amazingly modern. Following is an outline: Put the surface in general position in $\mathbb{R}^{3}$, and cut it open along carefully chosen horizontal planes. (His illustration is shown above.) Then each of the resulting connected pieces has either one, two, or three boundary curves: it is either a 2-cell, an annulus, or a "pair of pants".

More generally, let $E_{k}$ denote a region in the 2-sphere with $k$ boundary curves.

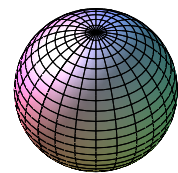

$E_{0}$

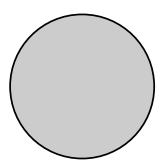

$E_{1}$

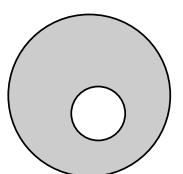

$E_{2}$

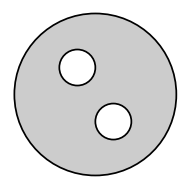

$E_{3} \quad \cdots$

Thus the Möbius construction splits the surface $\mathcal{F}$ into a disjoint union

$$
\mathbf{E}=E_{k_{1}} \sqcup E_{k_{2}} \sqcup \cdots \sqcup E_{k_{N}} \quad \text { with } \quad k_{j} \in\{1,2,3\} .
$$

Here is an example:
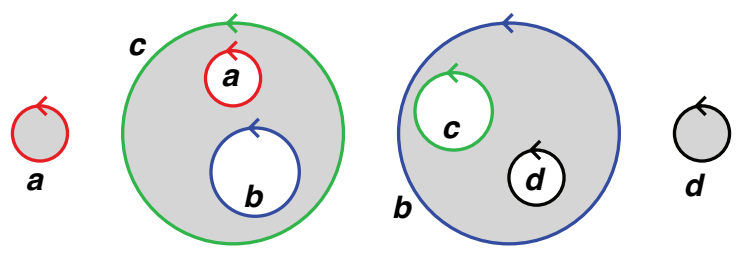

To get the original surface $\mathcal{F}$ we must identify each boundary curve of $\mathbf{E}$ with some other boundary curve, under a prescribed diffeomorphism. 
Lemma. If we simplify $E_{k} \sqcup E_{\ell}$ by identifying one boundary curve of $E_{k}$ with one boundary curve of $E_{\ell}$, then the result is diffeomorphic to $E_{k+\ell-2}$.

The proof is not difficult. Using this statement, we can simplify the set

$$
\mathbf{E}=E_{k_{1}} \sqcup \cdots \sqcup E_{k_{N}}
$$

inductively by identifying one pair of boundary curves at a time. After $N-1$ such identifications, we must obtain a connected set of the form $E_{2 p}$. (Here $p$ is Riemann's invariant, the genus.) As an example, in the figure above if we move the disks labeled a, d, and b into the corresponding holes, after shrinking in the last case, we are left with a region of type $E_{2}$.

Now each further identification of two curves amounts to adding a "handle" to the 2-sphere $S^{2}=E_{0}$. This completes the Möbius proof.

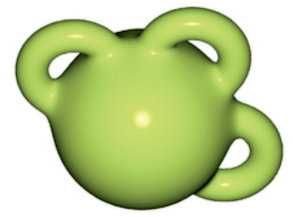

Example: A sphere with $p=3$ handles attached.

\subsection{Walther Dyck, Munich, 1888.}

Dyck (later ennobled as von Dyck) was probably the first to provide a clear definition of what topology is all about:

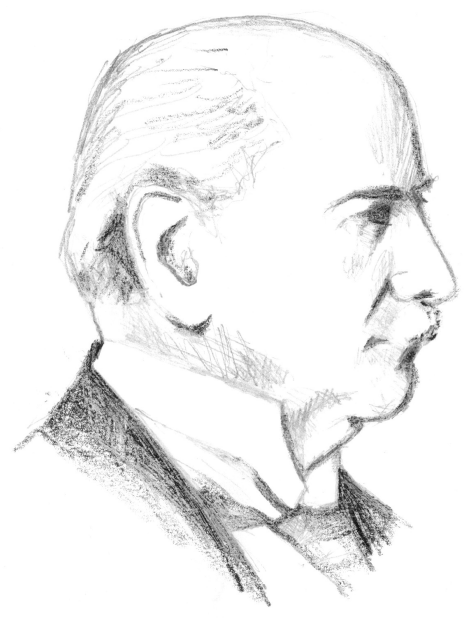

Topology is the study of properties which are invariant under continuous functions with continuous inverse.

He was also probably the first to state the Gauss-Bonnet Formula as a global theorem:

For any smooth closed two-dimensional manifold,

$$
\chi(M)=\frac{1}{2 \pi} \iint K d A .
$$


If the Gaussian curvature $K$ has constant sign, then it follows as an immediate corollary that the Euler characteristic has the same sign:

$$
\begin{aligned}
& \text { If } K>0 \text {, or } K=0 \text {, or } K<0 \text { everywhere, then } \chi>0 \text {, or } \\
& \chi=0 \text {, or } \chi<0 \text {, respectively. }
\end{aligned}
$$

This has served as the inspiration for every subsequent study relating curvature and topology.

\subsection{Henri Poincaré, Paris, between 1881 and 1907.}

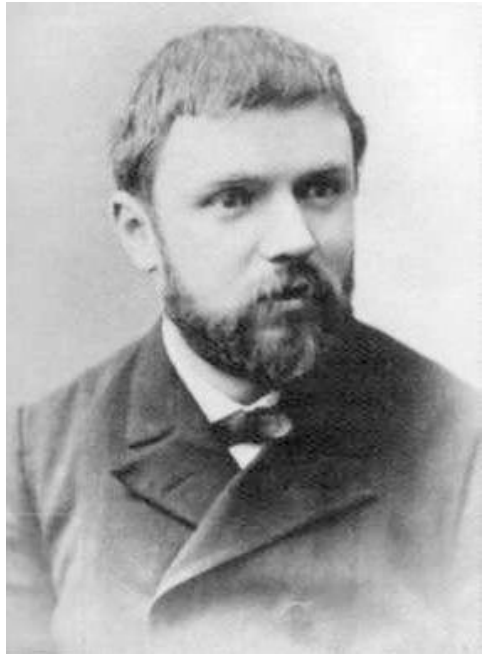

Poincaré can safely be described as the founder of modern topology. He sketched the theory of homology and Betti numbers, and described the Poincaré duality theorem. Furthermore he introduced the concept of homotopy, and defined the fundamental group, and the related concept of covering space. Particularly important for the study of surfaces was his description of the uniformization theorem for Riemann surfaces. Many details of these theories remained to be filled out, but he provided the fundamental outline.

\subsection{Paul Koebe, Berlin, 1907.}

Koebe and Poincaré proved the Uniformization Theorem at about the same time. Here is the statement.

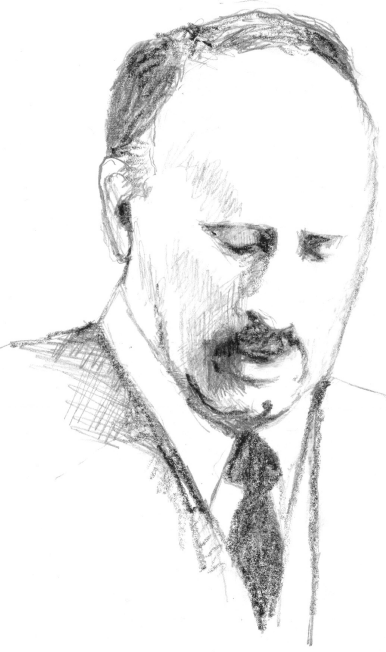

Theorem: The universal covering space $\widetilde{\mathcal{F}}$ of any Riemann surface $\mathcal{F}$ is conformally isomorphic to either:

(1) the Riemann sphere $\mathbb{C} \cup \infty$,

(2) the complex plane $\mathbb{C}$, or to

(3) the open unit disk $\mathbb{D} \subset \mathbb{C}$.

As an easy consequence we obtain the following. 
Corollary: Any Riemann surface has a metric of constant curvature:

- $K \equiv+1$ in Case (1),

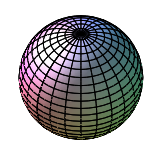

- $K \equiv 0$ in Case (2),

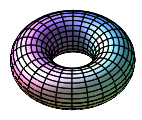

- $K \equiv-1$ in Case (3).

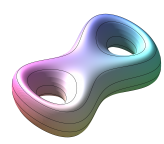

\subsection{Hermann Weyl, Göttingen, 1913.}

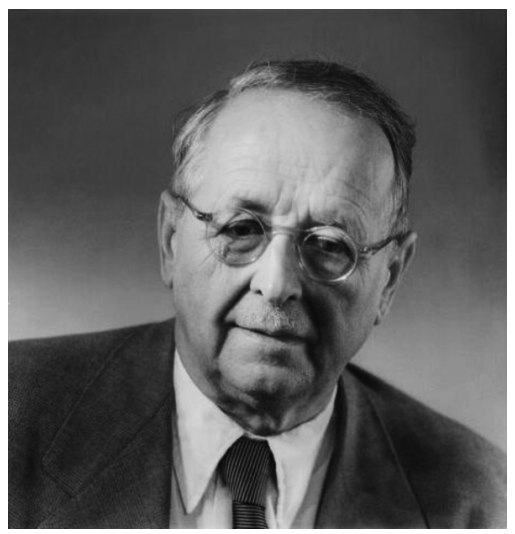

Weyl's book "Die Idee der Riemannschen Fläche" was an important turning point. Until its publication, the definition of Riemann surface had been rather vague, starting perhaps with a locally defined holomorphic function and following it through all possible analytic continuations. Weyl provided a clear definition, in terms of a topological surface with overlapping coordinate charts. This has provided a model for all subsequent work on complex manifolds and also on smooth manifolds.

\subsection{Tibor Radó, Szeged, 1925.}

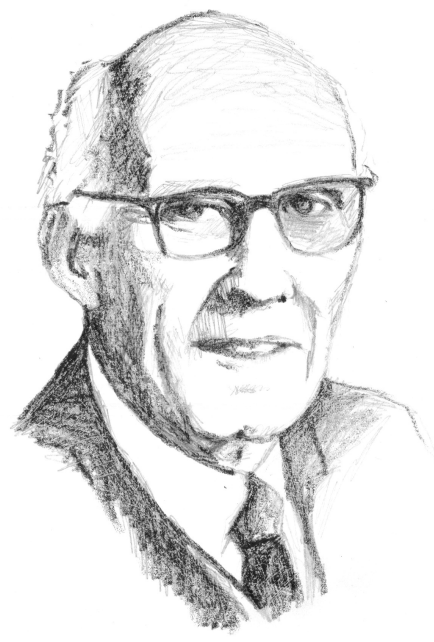

Radó completed the classification of compact oriented topological surfaces by showing that every such surface can be given the structure of a Riemann surface, as defined by Weyl. In particular, it can be given the structure of a smooth manifold. 
Ninety years later, the study of Riemann surfaces, and the closely related study of algebraic curves, remain flourishing fields of mathematics. Note, for example, that two of the four Fields Medals at the 2014 ICM were awarded for work in this area.

\section{Part 3. Three-Dimensional Manifolds}

The first description of a non-trivial 3-dimensional manifold surely occurred in the fourteenth century: the action of Dante's Divine Comedy took place in a world which is recognizably a topological 3 -sphere, with heaven at the upper pole and hell at the nether pole. However, the mathematical study of 3-dimensional manifolds began only in the late nineteenth century. To simplify the discussion, all manifolds are assumed to be orientable.

\subsection{Poul Heegaard, Copenhagen, 1898.}

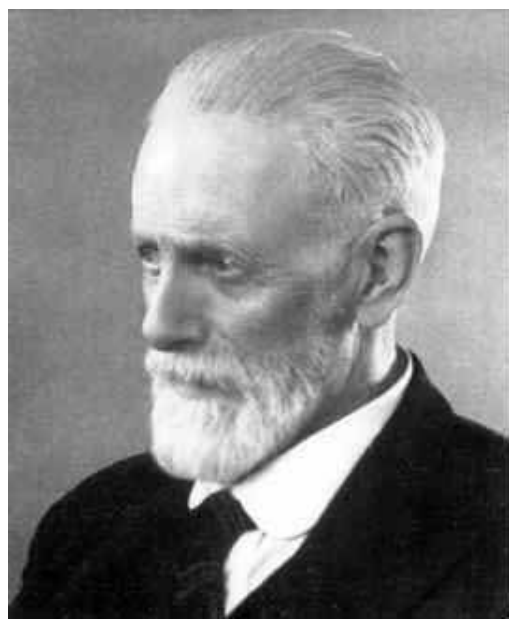

Heegaard showed that any closed orientable 3-manifold can be decomposed as a union of two handlebodies ${ }^{2}$ of the same genus, which intersect only along their boundaries. In other words, we can construct a model for any such manifold, by taking two copies of some handlebody $H$ and gluing their boundaries together under an arbitrary diffeomorphism.

Heegaard's result is very hard to apply, since the mapping class group, consisting of all isotopy classes of orientation preserving diffeomorphisms of a given surface, is very rich. However, it does provide an important technique for understanding general 3-manifolds. Incidentally, his theorem may have led to study of the mapping class group, which is an important object in its own right.

\subsection{Poincaré, Paris, 1904: The Poincaré Conjecture.}

This fundamental problem tormented topologists for the next hundred years:

\footnotetext{
${ }^{2}$ By definition, a handlebody of genus $g$ is the compact region in Euclidean 3-space obtained by adjoining $g$ disjoint "handles" to a 3-ball, where each handle is homeomorphic to $D^{2} \times[0,1]$, and intersects the 3 -ball precisely in $D^{2} \times\{0,1\}$. Thus the boundary of the handlebody is a surface of genus $g$.
} 


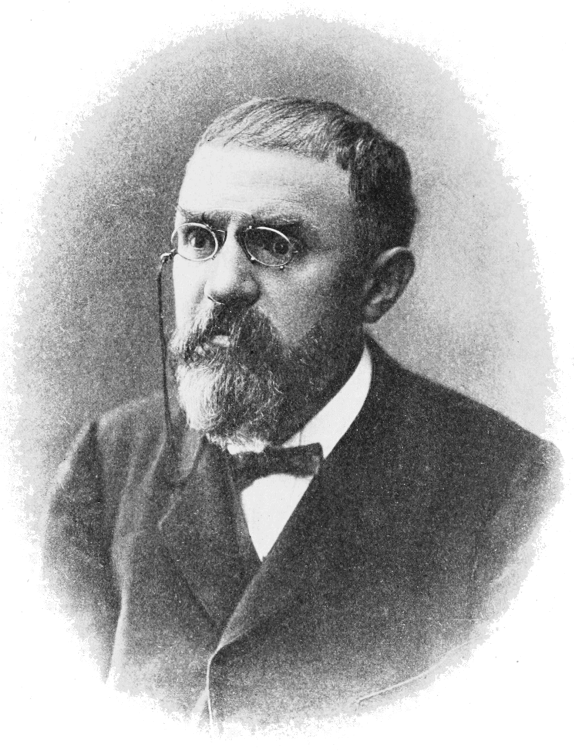

Question: If a closed threedimensional manifold has trivial fundamental group, is it necessarily homeomorphic to the standard sphere $S^{3}$ ?

Actually, Poincaré had originally conjectured in 1900 that any manifold with the homology of a sphere is homeomorphic to the standard sphere. However in 1904, using Heegaard's methods, he discovered a counterexample. His example, a smooth 3 -manifold with finite fundamental group of order 120, can most easily be described as the coset space $\mathrm{SO}_{3} / I_{60}$, where $I_{60}$ (the smallest non-Abelian simple group) is the group of rotations of 3-space which carry a regular icosahedron onto itself.

\subsection{James W. Alexander, Princeton, 1920s.}
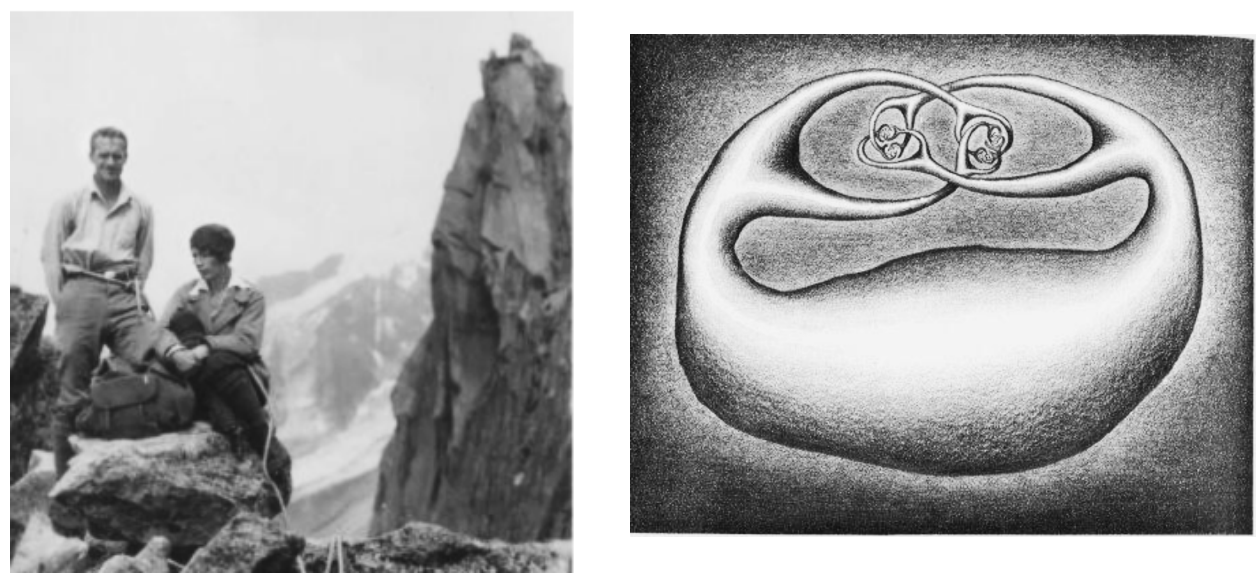

On the left, Alexander and his wife Natalie; Alexander horned sphere on the right. 
The Alexander duality theorem states that the homology of the complement of a complex $\mathbf{K} \subset S^{n}$ can be computed from the homology of $\mathbf{K}$. It can be thought of as a far reaching generalization of the Jordan curve theorem. For example, it implies that any closed connected $(n-1)$-manifold embedded in the $n$-sphere divides the sphere into two connected components. In the special case of a PL-embedded 2-sphere in $S^{3}$, he showed that the closure of each component of the complement is actually a PL copy of the three-dimensional closed ball. Similarly, for a PLembedded torus in $S^{3}$, at least one of the two complementary components must be a solid torus. On the other hand, his horned sphere example, illustrated above, showed that these last statements are not true for an arbitrary topological embedding. He also defined the Alexander polynomial of a knot, which is a fundamental invariant. $^{3}$

\subsection{Hellmuth Kneser, Greifswald, 1929.}
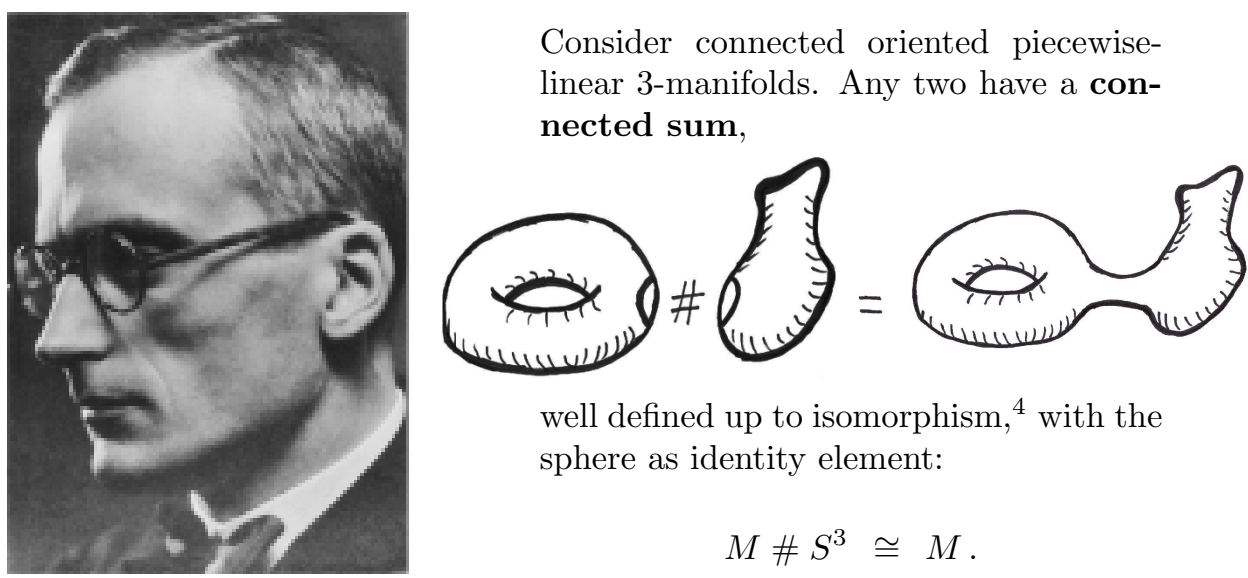

well defined up to isomorphism, ${ }^{4}$ with the sphere as identity element:

$$
M \# S^{3} \cong M
$$

Definition: The manifold $M \nsubseteq S^{3}$ is "prime" if this is the only way of expressing $M$ as a connected sum.

Theorem: Every compact $M$ is isomorphic to a connected sum

$$
M \cong P_{1} \# \cdots \# P_{k}
$$

of prime 3-manifolds.

(Many years later, I was able to complete this result by showing that this decomposition into primes is unique up to order and up to isomorphism. See MiLnoR [1962].)

\footnotetext{
${ }^{3}$ See AleXANDER 1922, 1924, 1928.

${ }^{4}$ Here I am using the word "isomorphism" to mean orientation preserving PL (= piecewiselinear) homeomorphism.
} 


\subsection{Herbert Seifert, Leipzig, 1933.}

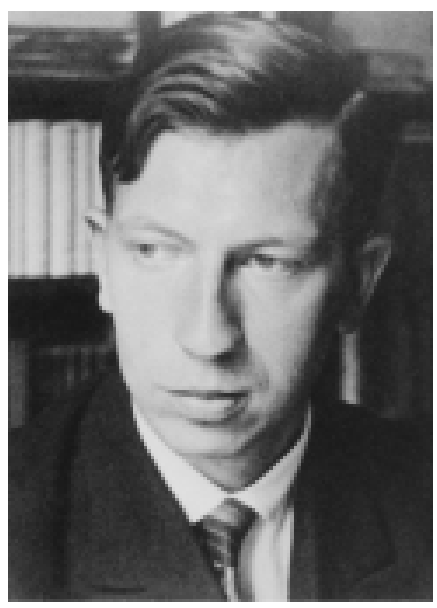

The Seifert fiber spaces form an important class of 3-dimensional manifolds that are well understood. They consist of manifolds which fiber over some surface, with the circle as fiber, where finitely many carefully restricted singular fibers are allowed.

3.6. Edwin Moise, University of Michigan, 1952.

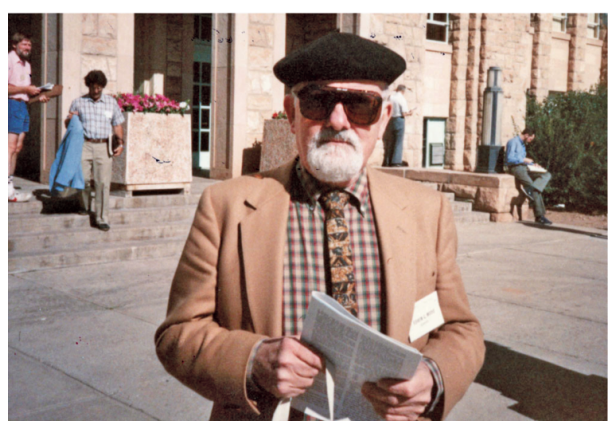

Moise (with a great deal of work) proved that every compact 3-manifold can be triangulated, and that this triangulation is unique up to PL-homeomorphism.

\subsection{Christos Papakyriakopoulos, Princeton, 1957.}

The first important breakthrough in 3-dimensional topology came with the proof of "Dehn's Lemma", which had been claimed, but not correctly proved, by Max Dehn in 1910:

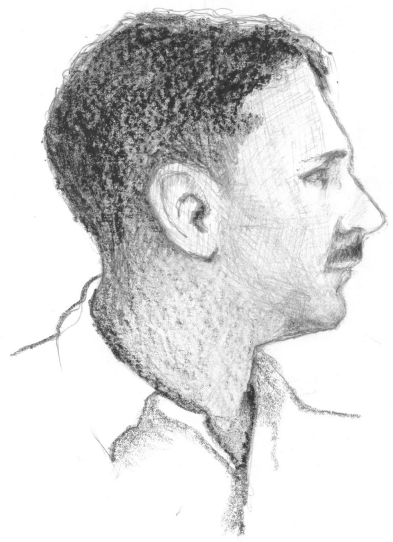

Theorem. Given a PL-map $f$ from the closed unit square $I^{2}$ into $\mathbb{R}^{3}$ such that $f^{-1} \circ f$ is single valued near the boundary, there exists a $P L$-embedding which coincides with $f$ near the boundary. 
The Papakyriakopoulos proof used an ingenious "tower" construction which can be outlined as follows. A regular neighborhood $N$ of the image $f\left(I^{2}\right)$ is a 3-manifold with boundary. If some boundary component has non-zero genus, then we can pass to some covering space of $N$, and the singular disk will lift to a singular disk in this covering space. The key step is to show that this lifted disk has simpler singularities so that, after repeating this construction finitely many times, we must arrive at the genus zero case. The proof can then be easily completed.

One important corollary is the following statement:

Let $\mathcal{K} \subset \mathbb{R}^{3}$ be a simple closed $P L$-curve. Then

$\mathcal{K}$ is unknotted if and only if $\pi_{1}\left(\mathbb{R}^{3} \backslash \mathcal{K}\right) \cong \mathbb{Z}$.

In fact if $\mathcal{K}$ is knotted, then $\pi_{1}\left(\mathbb{R}^{3} \backslash \mathcal{K}\right)$ contains a $\mathbb{Z} \oplus \mathbb{Z}$ subgroup, coming from the boundary of a tubular neighborhood of $\mathcal{K}$.

\subsection{Wolfgang Haken, Munich, and Friedhelm Waldhausen, Bonn, $1960 \mathrm{~s}$}
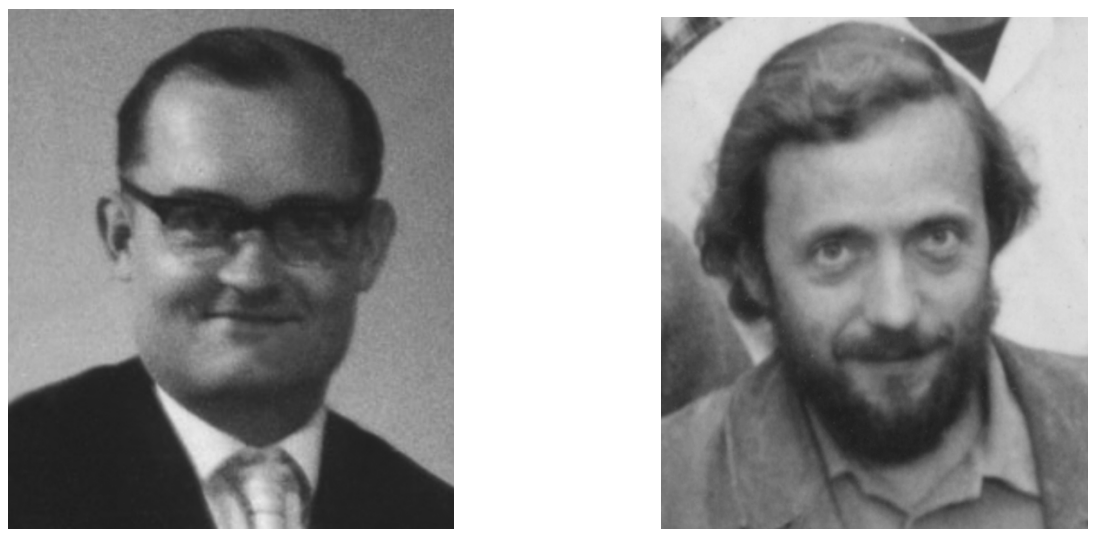

By definition, an incompressible surface in a compact orientable PL 3-manifold $M$ is a compact orientable PL-embedded surface such that:

- the fundamental group $\pi_{1}(\mathcal{F})$ is non-trivial and injects into $\pi_{1}(M)$, and

- if $\mathcal{F}$ has a boundary, then $M$ must also have a boundary, with $\partial \mathcal{F} \subset \partial M$.

An irreducible manifold with such an incompressible surface is called "sufficiently large", or briefly a Haken manifold. (Here the word irreducible means that any embedded 2-sphere bounds a 3-disk.) Given just one incompressible surface and cutting along it, Haken, in 1962, showed that one can inductively construct a sequence of incompressible surfaces that decompose the manifold into simply connected pieces. He never published the second part of this paper, which would have contained many details of his argument. However, Waldhausen in 1968 published a complete exposition, with further important results. In particular, he proved that any closed Haken manifold is uniquely determined, up to PL-homeomorphism, by its fundamental group. (In the case of a Haken manifold with boundary, one must also take account of the subgroups corresponding to boundary components.) Later GORDON AND LUECKE [1989] made use of this work in proving that a prime knot is uniquely determined by the fundamental group of its complement. 


\subsection{George D. Mostow, Yale, 1968.}

The next important contribution comes from a completely different area of mathematics.

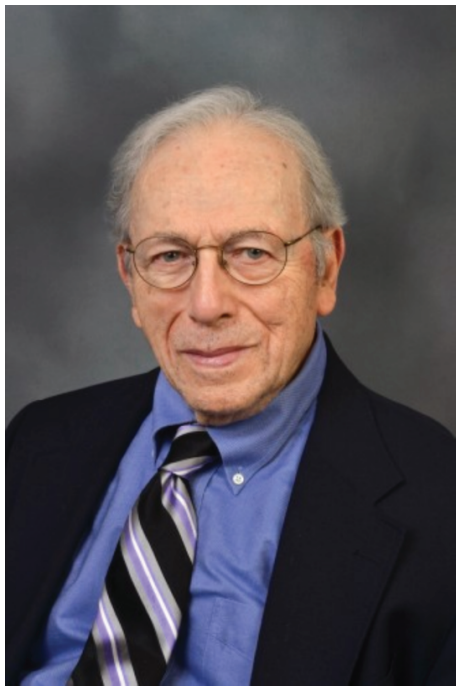

\begin{abstract}
Rigidity Theorem: A closed Riemannian manifold of dimension $\geq 3$ with curvature $K \equiv-1$ is uniquely determined up to isometry by its fundamental group.
\end{abstract}

This result was also proved by Margulis. It was extended to complete manifolds of finite volume by Prasad.

As an important corollary:

The volume of such a manifold is a topological invariant.

This was a completely new kind of topological invariant, unlike anything which had been known before. There are many other isometry invariants which are now promoted to homotopy type invariants, for example the lengths of closed geodesics and the eigenvalues of the Laplacian. However, the volume is a particularly convenient invariant to work with.

In the 1970s Robert Riley, a PhD student in Southampton, England, was studying representations of knot groups into the group $\operatorname{PSL}(2, \mathbb{C})$ of automorphisms of hyperbolic 3 -space, concentrating on those which map a meridian and parallel around the knot into parabolic group elements. He was able to find several examples (including the figure-eight knot) for which such representations not only mapped $\pi_{1}\left(S^{3} \backslash \mathcal{K}\right)$ isomorphically onto a subgroup $\Pi \subset \operatorname{PSL}(2, \mathbb{C})$, but could actually be promoted to homeomorphisms from the knot complement onto the quotient $H^{3} / \Pi$, which is a hyperbolic manifold of finite volume; that is, it is complete under a metric of constant negative curvature and of finite volume.

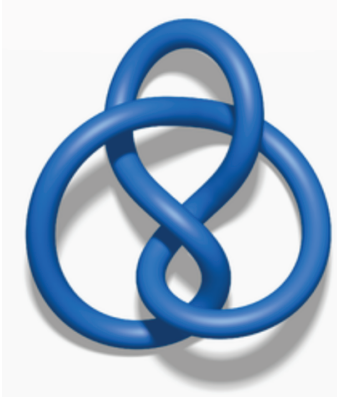

Thus the complement of the figure-eight knot in $S^{3}$ can be given a complete hyperbolic structure of finite volume.

Also in the 1970s, Troels Jørgensen at Columbia University found examples of subgroups

$$
\Pi \subset \operatorname{PSL}(2, \mathbb{C})
$$

such that the quotient $H^{3} / \Pi$ is a compact hyperbolic manifold which fibers over the circle. 


\subsection{William Thurston, Princeton, late 1970s.}

Thurston found many more hyperbolic knot complements.

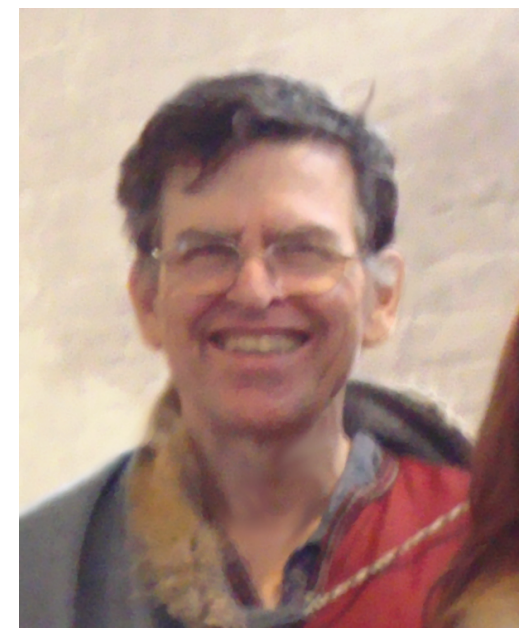

He computed the volume of the figureeight complement by "triangulating" it into two regular ideal 3-simplexes. (Compare GIESEKING 1912].) Using methods going back to Lobachevsky, this volume can be computed as

$V=-6 \int_{0}^{\pi / 3} \log |2 \sin (u)| d u=2.02988 \cdots$.

His main result about hyperbolic volumes can be stated as follows.

Theorem: The set of all volumes of hyperbolic 3-manifolds is well ordered. That is, any non-empty subset has a smallest element. Furthermore, there are at most finitely many non-homeomorphic manifolds for each fixed volume.

In fact, the volume of any manifold $M$ with $k$ ends, $k \geq 1$, is an increasing limit of volumes of manifolds with $k-1$ ends. The idea is that each "end" of $M$ can be identified with an embedded copy of $S^{1} \times S^{1} \times[0, \infty)$ which can be cut off and replaced by a solid torus $S^{1} \times \mathbb{D}$ in infinitely many different ways. Almost all of these simplified manifolds can be given a hyperbolic structure, and their volumes increase towards the volume of the original manifold.

As an example, the complement of the Whitehead link in $S^{3}$ has two ends, corresponding to the two components of the link. Either or both of the interlinked solid tori in the figure can be emptied and then filled in with a new solid torus in infinitely many ways. In this particular case, the link complement has hyper-

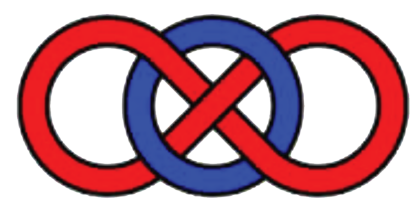
bolic volume $V=3.66386 \cdots$. 
3.11. William Jaco, Peter Shalen, and Klaus Johannson, late 1970s.
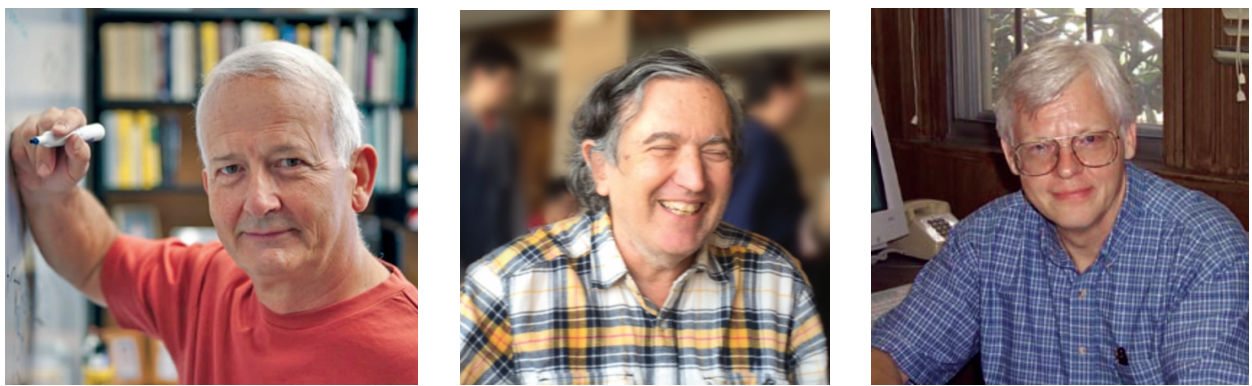

The JSJ-decomposition, named after these three 5 is a way of splitting up a 3 -manifold into simpler pieces by cutting along embedded spheres and tori. Here is one statement of it.

Theorem. Any irreducible orientable closed 3-manifold has a unique (up to isotopy) minimal collection of disjointly embedded incompressible tori such that each component of the 3-manifold obtained by cutting along the tori is either atoroidal or Seifertfibered.

Here the word atoroidal means that there is no incompressible embedded torus.

\subsection{Thurston, 1982: The Geometrization Conjecture.}

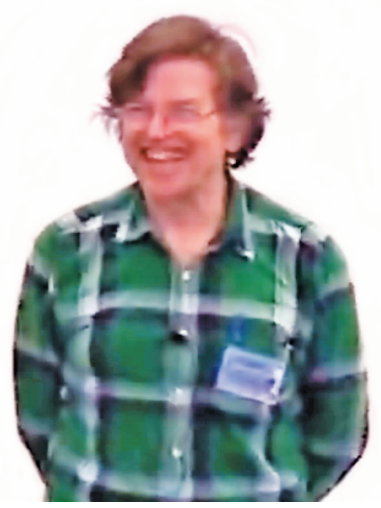

This very bold conjecture proposed that every closed 3-manifold can be built up out of pieces which have a simple geometric structure. More explicitly, it asserts that every smooth closed 3-manifold can be decomposed, by embedded spheres and tori, into manifolds $M_{j}$, each of which can be given a locally homogeneous structure, so that the universal covering $\widetilde{M}_{j}$ is a homogeneous space.

Furthermore, there are exactly eight possibilities for $\widetilde{M}_{j}$.

Three of these are the three classical geometries:

(1) The Sphere $S^{3}$, with curvature $K \equiv+1$.

Riemannian manifolds with $S^{3}$ as universal covering had been classified by Heinz Hopf in 1925 .

\footnotetext{
5 Jaco, Shalen, and Johannson were respectively in Stillwater, Oklahoma, in Chicago, and in Bielefeld, Germany.
} 
(2) The Euclidean space $\mathbb{R}^{3}$, with curvature $K \equiv 0$. The corresponding compact flat manifolds had been classified by Bieberbach in 1911.

(3) The Hyperbolic space $H^{3}$, with $K \equiv-1$.

This is the most interesting and difficult case.

The next two geometries are fairly easy to understand.

(4) $\widetilde{M} \cong \mathbb{R} \times S^{2}$. Example: $M=S^{1} \times S^{2}$.

(5) $\widetilde{M} \cong \mathbb{R} \times H^{2}$. Example: $M=S^{1} \times$ (hyperbolic surface) .

For the last three geometries, $\widetilde{M}$ will be a three-dimensional Lie group with a maximally symmetric left invariant metric.

(6) Nilgeometry, with nilpotent group $\left(\begin{array}{lll}1 & x & y \\ 0 & 1 & z \\ 0 & 0 & 1\end{array}\right)$.

Example: A non-trivial circle bundle over the torus.

(7) Solvgeometry, with solvable group $\left(\begin{array}{ccc}1 & 0 & 0 \\ x & e^{z} & 0 \\ y & 0 & e^{-z}\end{array}\right)$.

Example: Most torus bundles over the circle.

(8) $\widetilde{\mathrm{SL}}(2, \mathbb{R})$ geometry.

Example: The unit tangent bundle of a hyperbolic surface.

Note that the Geometrization Conjecture includes the Poincaré Conjecture as just one special case.

Thurston managed to prove this Geometrization Conjecture in many interesting and difficult cases. However the general case, and in particular the Poincaré Conjecture, eluded him.

\subsection{Richard Hamilton, Cornell University, 1982.}

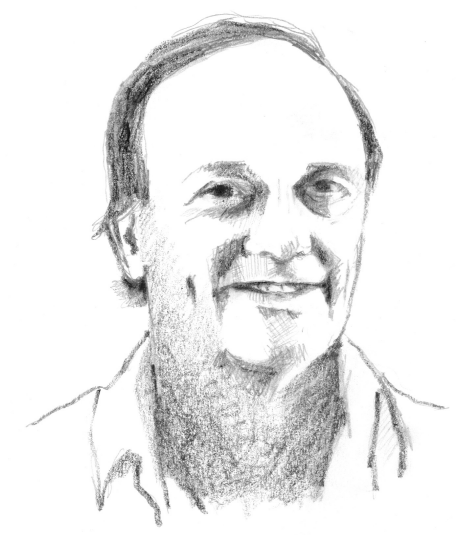

Hamilton introduced a completely new method for studying manifold topology, by means of his Ricci flow differential equation

$$
\frac{\partial g_{j k}}{\partial t}=-2 R_{j k}
$$


Here $g_{j k}$ is the metric tensor on a local coordinate chart for a closed Riemannian manifold, and $R_{j k}$ is the associated Ricci curvature tensor. Under the somewhat analogous heat equation, heat will flow from hot regions to cold regions, so as to converge towards constant temperature. Similarly, under Ricci flow, the intuitive idea was that curvature should flow from positively curved regions towards negatively curved regions, so as to converge towards a uniform distribution of curvature.

If we start with a manifold having strictly positive Ricci curvature, then Hamilton was able to prove that this really works. In this case, the metric flows towards a metric of constant positive curvature, proving the the manifold is diffeomorphic to the standard 3-sphere. But for more general initial conditions, the metric will develop complicated singularities, and Hamilton was unable to make further progress.

\subsection{Grigori Pereleman, St. Petersburg, 2003.}

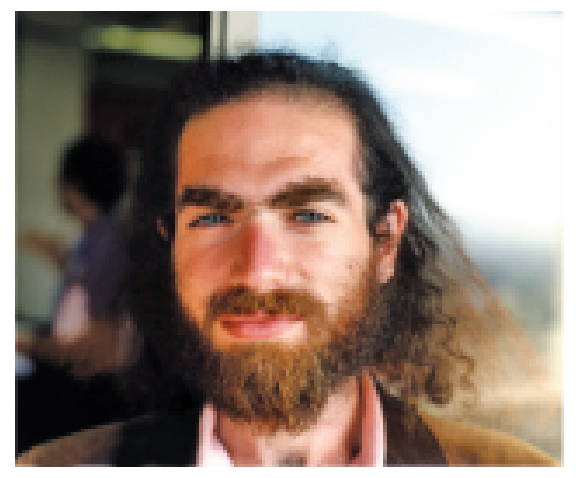

By a careful and ingenious analysis of the singularities which develop during Ricci flow, Perelman was able to resolve all of the problems which Hamilton encountered.

Some singularities were relatively tame and could be eliminated. Others corresponded to shrinking an embedded sphere to a point, so as to yield a direct sum decomposition, while still other singularities corresponded to a torus decomposition. Finally, in the absence of singularities, the flow must lead towards a homogeneous limit. In this way, he was able to complete a proof of the full Geometrization Conjecture, including the Poincaré Conjecture as one special case.

\section{Part 4. Four-Dimensional Manifolds}

Algebraic varieties of complex dimension two, hence real dimension four, were studied for example by PICARD AND SIMART 1897, 1906], POINCARÉ [1904], ENRIQUES [1905], and LEFSCHETZ [1924]. Physicists were interested in a different kind of 4-manifold: those which might serve as a model for the space-time universe. (See FRIEDMAN 1922.) However, except for a few remarks in SEIFERT AND THRELFALL [1934], serious topological studies of general 4-manifolds began only in the 1950s. 
At that time, it was generally expected that the topology of $n$-dimensional manifolds would become more and more difficult as $n$ increased. This certainly turned out to be true up to dimension four 6

\subsection{A. A. Markov Jr., Moscow, 1958.}

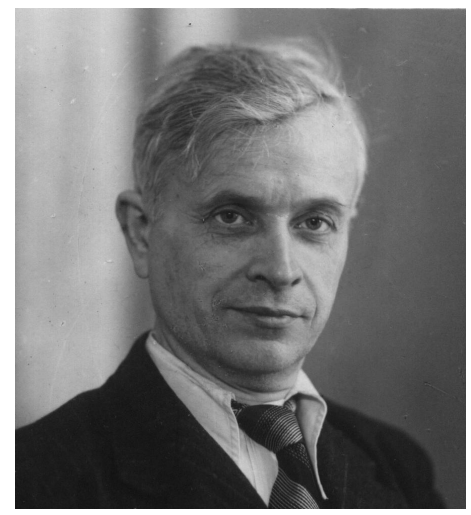

Markov made a devastating contribution to the study of 4-dimensional manifolds:

Theorem. The problem of classifying closed $n$-manifolds up to homeomorphism is algorithmically unsolvable for $n \geq 4$.

Here is an outline of his proof, taking $n=4$ for simplicity.

Given any finite group presentation $P$ with $p$ generators and $q$ relators, construct an associated 4-dimensional manifold $M(P)$ as follows 7 Start with the connected sum of $p$ copies of $S^{1} \times S^{3}$. Perform a surgery by boring out $q$ disjoint copies of $S^{1} \times D^{3}$, representing the $q$ relators, and fill each one with a copy of $D^{2} \times S^{2}$, so as to kill the corresponding element of the fundamental group. Now let $P^{\prime}$ be the presentation obtained from $P$ by adding $p$ copies of the trivial relator "1". Markov showed that $M\left(P^{\prime}\right)$ is homeomorphic to the connected sum of $q$ copies of $S^{2} \times S^{2}$ if and only if the associated group is trivial. Since the triviality problem for finitely presented groups is known to be algorithmically unsolvable (see ADYAN [1955]), the conclusion follows.

Thus, for any hope of a classification theorem for 4-manifolds, we must consider only manifolds with known fundamental group.

\footnotetext{
${ }^{6}$ In fact higher dimensions are actually easier to work with. See for example SMALE 1961], which proved a version of the Generalized Poincaré Conjecture in higher dimensions.

${ }^{7}$ Compare the exercises at the end of Chapter 7 in SEIFERT AND Threlfald [1934].
} 


\subsection{J. H. C. Whitehead, Oxford, 1949.}

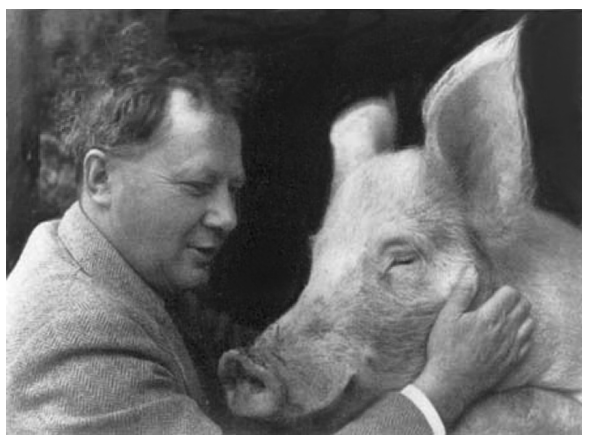

Nine years earlier, Whitehead had classified simply connected fourdimensional complexes up to homotopy type.

Applied to manifolds, his result has the following consequence. (Compare MILNOR 1958].)

Corollary. A closed oriented simply connected 4-manifold $M$ is determined, up to oriented homotopy type, by its intersection form

$$
H_{2}(M) \otimes H_{2}(M) \rightarrow \mathbb{Z}
$$

This form is symmetric, bilinear, and unimodular (that is, its determinant is \pm 1 ).

The classification of such symmetric bilinear forms is an important and non-trivial problem in number theory. The classification is easy in the case of indefinite forms, but very difficult in the positive definite case, since the number of distinct forms grows very rapidly with the rank.

As an example, it follows from work of Carl Ludwig Siegel that there are more than 904,000,000 distinct positive definite unimodular forms of rank equal to $\mathbf{3 0}$.

\subsection{Vladimir Rokhlin, Moscow, 1952.}

The following result was a very important step towards understanding higher dimensional manifolds and towards the beginnings of Differential Topology.

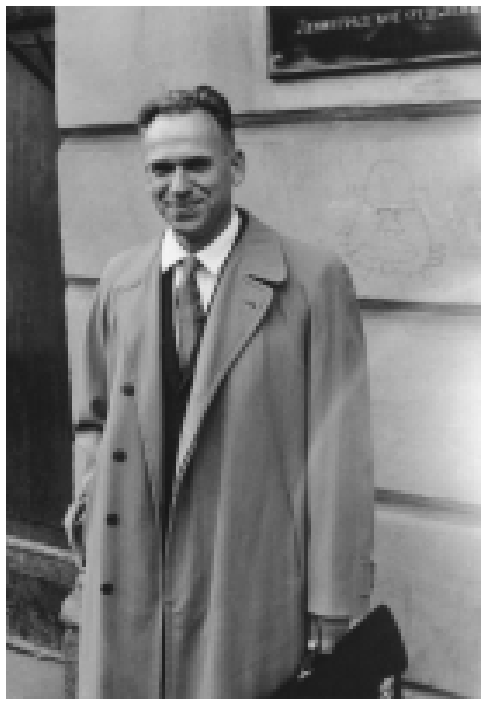

Theorem. If a smooth closed 4-manifold has positive definite intersection form, and if the self-intersection $u \cdot u \geq 0$ takes only even values, then the rank of this form (that is, the middle Betti number) must be divisible by 16.

By way of contrast, the rank of an arbitrary positive definite unimodular form which takes only even values can be any multiple of 8 . The simplest non-trivial example can be described by the $E_{8}$ Dynkin diagram which is shown below. 


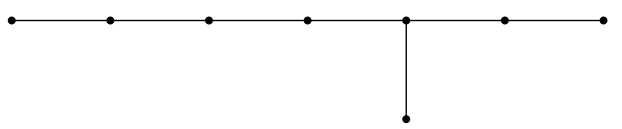

Dynkin diagram

Here each dot stands for a basis vector with self-intersection $u \cdot u=2$, where two distinct basis vectors have intersection number $u \cdot v$ equal to +1 if they are joined by a line segment, and zero otherwise. Thus no smooth closed 4 -manifold can have this symmetric bilinear form as its intersection form.

At the time, the restriction to smooth manifolds seemed like a minor technicality, but it has turned out to be crucial.

\subsection{Michael Freedman, University of California San Diego, 1982.}

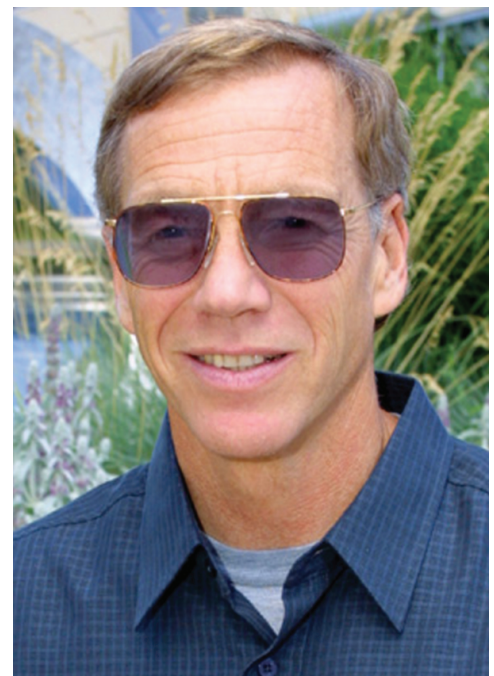

In particular, it follows that there are many closed topological 4-manifolds which have an even positive definite quadratic form of rank congruent to 8 modulo 16. According to Rokhlin, such manifolds $M$ do not admit any smooth structure in the following very strong sense:

\section{No 4-manifold with the same homotopy type as $M \quad$ admits a differen- tiable structure.}

Freedman's proof was based on wildly non-differentiable methods, including the concept of a "grope", as illustrated here. (The concept, and this illustration, are due to CANNON [1978].)
Theorem. A closed oriented simply connected topological 4-manifold is uniquely determined, up to homeomorphism, by

- its intersection form, and

- its "Kirby-Siebenmann invariant." This is an element of $\mathbb{Z} / 2 \mathbb{Z}$, which is always zero in the case of a smooth manifold.

Furthermore, any symmetric unimodular bilinear form can be realized by a topological manifold.

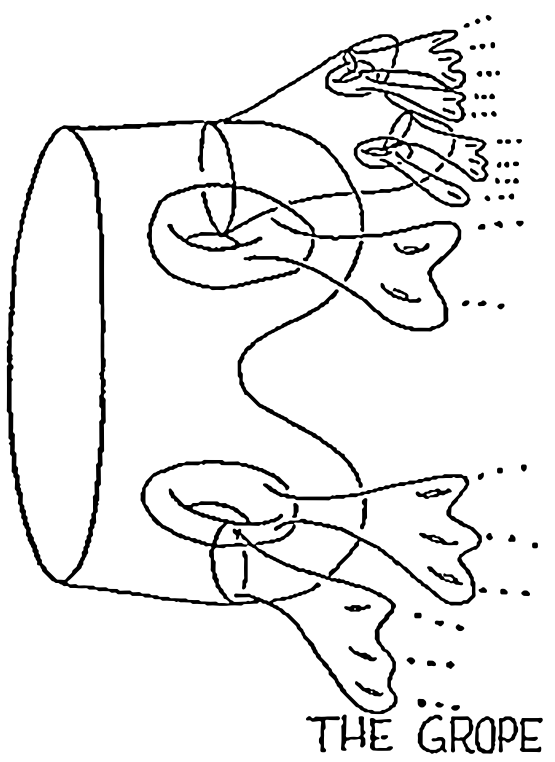




\subsection{Simon Donaldson, Oxford, 1983.}

One year later, using completely different methods, Donaldson proved an equally amazing result.

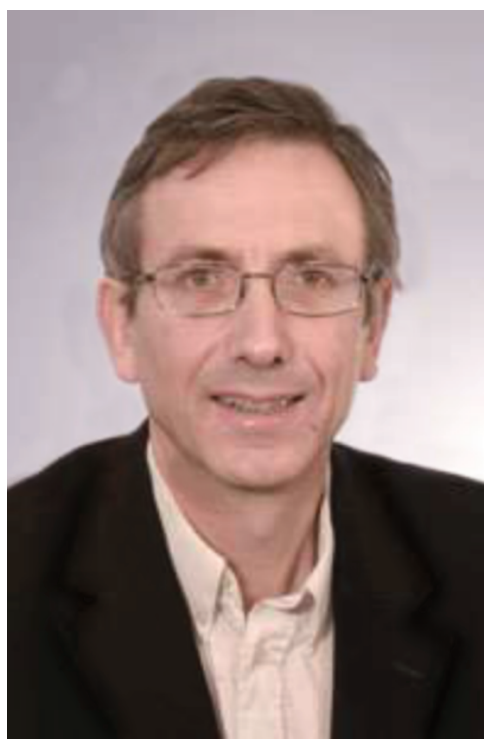

Theorem. If a smooth, closed simply connected 4-manifold $M$ has positive definite intersection form, then this form is diagonalizable.

Hence, by Freedman's theorem, $M$ must be homeomorphic to a connected sum

$$
\mathbb{C P}^{2} \# \cdots \# \mathbb{C P}^{2}
$$

To illustrate the striking contrast between these two results, note the following:

Corollary. Among the more than 904,000,000 homeomorphism classes of topological manifolds with positive definite intersection form of rank 30, only one is represented by a smooth manifold.

Donaldson's proof is based on the study of "instantons", inspired by work in mathematical physics. It uses very little topology but a great deal of deep analysis.

\subsection{Clifford Taubes, Harvard, 1987.}

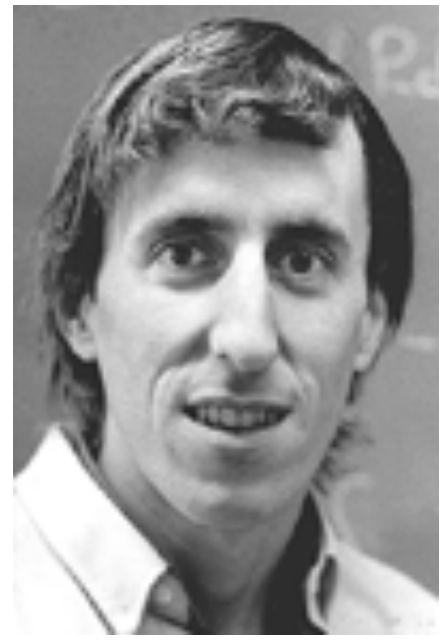

A number of mathematicians noticed that the combination of Freedman's hard-core topology and Donaldson's analytic methods had even more amazing consequences. (Compare GoMPF 1983, 1993.) Here is one example, due to Taubes:

Theorem. The Euclidean space $\mathbb{R}^{4}$ can be given uncountably many distinct differentiable structures. 
By way of contrast, for every $n \neq 4$, the space $\mathbb{R}^{n}$ has only one differentiable structure up to diffeomorphism 8

Thus dimension four is really different from all other dimensions!

\subsection{Conclusion: What Next?}

There is still a great deal to be learned about smooth 4-manifolds. The smooth Poincaré Conjecture in dimension four is a particularly tantalizing open problem. (Compare Freedman, Gompf, Morrison And Walker [2010].) To put it in context, consider what is known in other dimensions:

For any $n \geq 1$, it is easy to see that the set of oriented diffeomorphism classes of manifolds homeomorphic to the standard $n$-sphere forms a commutative associative semigroup $\mathscr{S}_{n}$ under the connected sum operation.

Theorem (Kervaire and Milnor). For $n \neq 4$, this semigroup $\mathscr{S}_{n}$ is actually a finite Abelian group.

However the semigroup $\mathscr{S}_{4}$ is completely unknown.

Is it trivial?

If not, is it at least a group?

How big is it?

If not a group, what kind of semigroup is it?

\section{Appendix: Further Comments on Various Sections}

A1.1. Euler. Although born and educated in Basel, Euler was unable to get a position there, and hence was happy to accept an offer from the Imperial Russian Academy of Sciences in St. Petersburg.

The city named Königsberg existed for 690 years. It was founded in 1255, when the original population, which spoke a Baltic language, was annihilated and replaced by German settlers. The German city ceased to exist in 1945 when it was taken over by Soviet forces and renamed as Kaliningrad. During the 19th and 20th centuries, Königsberg produced many mathematicians, including Rudolph Lipschitz, Alfred Clebsch, David Hilbert, Hermann Minkowski, and Jürgen Moser.

A1.2. Euler. In 1741, conditions in Russia had become unsettled. Hence Euler accepted a position at the Berlin Academy when it was offered by Frederick the Great.

As discussed in $\S 2.1$, the first person to consider the concept of "Euler characteristic" for non-convex polyhedra in 3-space was probably Simon L'Huilier. Johann

\footnotetext{
${ }^{8}$ This statement follows from STALLINGS 1962], together with the theory of obstructions to existence and uniqueness of smoothing which is due to J. Munkres, M. Hirsch, and B. Mazur. See MILNOR 2011] for further references.
} 
Listing in 1862 studied more general polyhedra, and Poincaré in 1895 was the first to suggest that the Euler characteristic of a manifold could be computed as an alternating sum of Betti numbers. (The Euler characteristic, defined in this way, is often called the Euler-Poincaré characteristic.) In fact, Poincaré's work on homology, as developed by many people in the early 20 th century, made it natural to consider the construction

cell complex $\mathbf{K} \quad \mapsto \quad$ chain complex $C_{*}(\mathbf{K}) \quad \mapsto \quad$ homology groups $H_{n}(\mathbf{K})$.

Using coefficients in a field, it was then not hard to show that the integer

$$
\chi(\mathbf{K})=\sum(-1)^{n} \operatorname{rank}\left(C_{n}(\mathbf{K})\right)
$$

is equal to the alternating sum of Betti numbers $\sum(-1)^{n} \operatorname{rank}\left(H_{n}(\mathbf{K})\right)$.

However, the proof that homology groups are topological invariants was much more difficult. A basic necessary tool, the simplicial approximation theorem, had been provided by L. E. J. Brouwer 9 However, Brouwer did not apply it to homology theory. The first proof of the topological invariance of Betti numbers by ALEXANDER [1915] seems rather fuzzy to me. (In his defense, Alexander was a fresh $\mathrm{PhD}$ at the time.) The situation became much clearer after topologists learned, under the influence of Emmy Noether, that they should be looking at homology groups, and that the Betti numbers should simply be defined as the ranks of these groups (see HiRzEBRUCH 1999). The first expositions that I find really readable are those of SEIFERT AND Threlfald [1934], and Alexandroff AND Hopf [1935].

A1.4. Gauss. In terms of the concept of degree (introduced much later), the Gauss integral computes the degree of the associated map $(x, y) \mapsto(x-y) /\|x-y\|$ from torus to sphere.

A2.1. L'Huilier. The paper, LhuILIER AND GERGONNE [1812-1813], is an abridgment by Gergonne of a longer manuscript which had been submitted by L'Huilier. Unfortunately, its description of the basic invariant $n$ (in our terminology the genus) is rather vague. (I have no way of knowing whether L'Huilier's original presentation was more precise.) Let me quote Gergonne's precise language (page 168), since I am not sure about my translation:

"En général un polyèdre terminé par une surface unique peut être percé, de part en part, par un nombre plus ou moins grand d'ouvertures distinctes. Si $n$ désigne le nombre de ses ouvertures, ..."

In any case, there is a clear recognition that there is such a number $n$ associated with any polyhedral surface, and a procedure for computing it. As one example, for the surface of a "ring-shaped" polyhedron, he states (also on page 168) that the Euler characteristic is zero.

The Académie de Genève, founded by Calvin in 1559, was called "Imperial" for a few years during the Napoleonic era, and has been known as the Université de Genève since 1873.

\footnotetext{
9 According to DiEUdONNÉ [1988], “... the epoch-making results of Brouwer in 1910-1912 ... may rightly be called the first proofs in algebraic topology, since Poincaré's papers can only be considered as blueprints for theorems to come."
} 
A2.2. Abel. During the year 1826, a stipend from the Norwegian government enabled Abel to visit several European countries. His stay in Berlin was particularly successful, and he published seven papers in the newly founded "Crelle's Journal". His stay in Paris was less successful. Perhaps his most important work, an addition theorem for algebraic integrals, was submitted to the French Academy, where it languished on Cauchy's desk for fifteen years. Back in Norway, after two years of intense mathematical activity but declining health, Abel died of tuberculosis at the age of 26. According to Charles Hermite:

"Abel has left mathematicians enough to keep them busy for five hundred years."

A2.3. Riemann. Although I have tried to explain the ideas in RIEMANN 1857, p. 97], I must admit that his presentation is very hard for me to follow in detail.

The first person to use the term genus in connection with Riemann surfaces was Clebsch [1865]. (Compare Hirzebruch AND KRECK [2009].) However, for Clebsch the genus of a Riemann surface was not an integer but rather an equivalence class containing the surface, in analogy with the use of the word in biology, or in the theory of quadratic forms. Thus for Clebsch the Riemann sphere belonged to the "first genus" (den ersten Geschlecht), while a torus or elliptic curve belonged to the "second genus," and so on. For the further history, let me refer to correspondence between Poincaré and Felix Klein in 1881. As described in GRAY, 2013, p. 230], Poincaré asked: What was the Geschlecht in the sense of analysis situs, and was it the same as the genre that he, Poincaré, had defined?

Klein replied that:

Genus in the sense of analysis situs is the maximal number of closed curves which can be drawn without disconnecting the surface, and is materially the same number as the genus of the algebraic equations defining the surface.

A2.4. Möbius. The jump from Riemann surfaces, perhaps in the complex projective plane, to smooth surfaces in Euclidean 3-space was a drastic one, which made the subject much more intuitive. However, it is not clear how it was justified. Today we would describe the Möbius argument as a proof by Morse Theory methods. Using partitions of unity, it is much easier to construct a Morse function than to construct an embedding in 3 -space. However, such methods were certainly not available at the time.

Note that the Möbius argument makes essential use of orientability. In fact both Riemann surfaces and closed surfaces embedded in 3-space are necessarily orientable. One would have to work a little harder to adapt the Möbius proof to non-orientable surfaces.

A3.1. Heegaard. Nowadays, it is customary to work with Heegaard splittings for piecewise linear manifolds. (See for example HEMPEL [1976].) However, one could equally well construct a Heegaard splitting of a smooth manifold, using a Smale type argument with "nice" Morse functions. Heegaard's original argument did use differentiable methods, but in a rather intuitive style. 
A3.2. Poincaré. A convenient model for Poincaré's non-trivial homology sphere is the spherical dodecahedral space, obtained from a regular dodecahedron by identifying each face with the opposite face under a translation composed with a rotation through $2 \pi / 10$. With a little care, the resulting space can be given a metric with constant positive curvature. (Compare SEIFERT AND THRELFALL [1934].) It is then not difficult to show that it is homeomorphic to the coset space $\mathrm{SO}_{3} / I_{60}$. This coset space can be described equivalently as the space with one point corresponding to each regular icosahedron (or dodecahedron) of unit size centered at the origin. For a proof that the dodecahedral space is equivalent to Poincaré's original construction, see CANNON [1978].

As in all 19th century mathematics, one has to be careful since the meanings of some words have changed. Thus for Poincaré, a space is "simply connected" if it is topologically a cell or sphere. His "Betti number" is our Betti number plus one.

A3.3. Alexander. Later, in the 1930s, Alexander was one of the founders of cohomology theory, defining cohomology groups for arbitrary compact metric spaces. (The Alexander cohomology groups are isomorphic to the Čech cohomology groups which were defined a few years later, although the construction is quite different.)

I never met Alexander, who was a virtual recluse from the time of his retirement from the Institute for Advanced Study in 1951 until his death twenty years later. Perhaps he wanted to stay out of sight since the political climate during the McCarthy era was very dangerous for someone with his left-wing political views. Alexander was a millionaire through inherited wealth, and never accepted a salary from the Institute.

A3.4. Kneser. If the manifold $M$ is not prime, then it can be described as a connected sum $M_{1} \# M_{2}$. Similarly, if one of these two manifolds is not prime, it can be expressed as a connected sum, and so on. The problem is to show that this construction must eventually stop. I was stuck trying to resolve this question some 50 years ago (see MILNOR 1962]), and was both relieved and chagrined to discover that Kneser had solved it before I was born.

Kneser, like Bieberbach, Teichmüller, and Witt, was an early supporter of the Nazi party.

A3.7. Papakyriakopoulos. I was in Princeton during much of the time Papakyriakopoulos was there, and certainly knew him, but can't remember ever interacting with him. Perhaps we were both very shy. He worked very much by himself, supported by a small stipend which Ralph Fox had managed to arrange. It was a complete surprise to me, and I think to many others, when he came up with such important results, including not only Dehn's Lemma, but also the loop theorem, which is a sharpened version of it, and the sphere theorem which asserts that for every orientable PL 3-manifold $M$ with $\pi_{2}(M) \neq 0$, one can find a PL-embedded sphere representing a non-trivial element of $\pi_{2}(M)$.

There is of course a long history of knot theory, both before and after Papakyriakopoulos, starting with attempts at a census by P. G. Tait in the 19th century, 
and continuing with the work of J. W. Alexander, K. Reidemeister, and many others. For more complete descriptions see for example, Crowell AND Fox [1963], ROLFSEN [1976], LiCKORISH [1997], and MANOLESCU [2014].

A3.10. Thurston. Compare Thurston [1980, 1982] as well as Gromov [1979-1980] and NEUMANN AND ZAGIER 1985].

It would be very interesting to know more about the number theory of the numbers which occur as volumes of hyperbolic 3-manifolds. (See for example BoREL [1981] and ZAGIER 1986], as well as my comments in THuRSTON [1980, Chapter 7].

It was always amazing to talk to Thurston. I was typically very skeptical about his mathematical claims, which often seemed quite wild. But he was never wrong.

A4.2. Whitehead. For further details, see WHITEHEAD 1949a and MILNOR [1958]. Whitehead was a good friend, and the only mathematician I ever met who raised pigs as a hobby.

A symmetric bilinear form $x, y \mapsto x \cdot y \in \mathbb{Z}$, where $x$ and $y$ range over a free Abelian group $\mathbb{Z}^{r}$, is often identified with its associated quadratic form $x \mapsto x \cdot x$. For surveys of such forms, see SERRE [1970] or Milnor AND HusEmolleR [1973]. By definition, two such forms belong to the same genus if they have the same rank and signature 10 so that they are isomorphic over the real numbers, and if they are also isomorphic modulo $n$ for every $n$. In the unimodular case, with fixed rank $r$ and signature $\sigma$, there are only two genera, depending whether the quadratic form is "even," taking only even values, or "odd," taking both odd and even values. These invariants are restricted only by the obvious condition that $|\sigma| \leq r$ with $\sigma \equiv r(\bmod 2)$, and by the not so obvious condition that $\sigma \equiv 0(\bmod 8)$ in the even case. In fact, in the indefinite unimodular case the genus is a complete isomorphism invariant. However, in the definite case, Carl Ludwig Siegel's analytic computation of "mass" of a genus yields a very useful lower bound for the number of distinct isomorphism classes 11 By definition, the mass is the sum, over all isomorphism classes $\Phi$ in the genus, of the ratio $1 /|\operatorname{Aut}(\Phi)|$, where $|\operatorname{Aut}(\Phi)| \geq 2$ is the order of the group of automorphisms of a representative quadratic form.

A4.3. Rokhlin 12 The original statement of Rokhlin's theorem (using modern notation) is that a 4-manifold with Stiefel-Whitney class $w_{2}=0$ must have Pontryagin class $p_{1}$ divisible by 48 . In fact the Pontryagin number $p_{1}\left[M^{4}\right]$ is equal to three times the signature. In the simply connected case, the intersection form is even if and only if $w_{2}=0$. His proof, based on relations between characteristic classes, cobordism, and homotopy groups of spheres in low dimensions, helped to inspire the new field of differential topology. (René Thom's full theory of cobordism was published two years later.) For further developments, see for example Kervaire AND MilnoR [1960] and HirzebruCH [1966, p. 199]. Rokhlin is also known for his contributions to ergodic theory.

\footnotetext{
${ }^{10}$ Every such form can be diagonalized over the real numbers. By definition the signature is the sum of the signs of the diagonal elements.

${ }^{11}$ André Weil described Siegel as "the greatest mathematician of the first half of the 20th century." Siegel was a conscientious objector who spent World War I imprisoned in a German psychiatric institute. He spent World War II in the United States.

${ }^{12}$ Rokhlin's name was formerly transliterated as Rohlin.
} 
Rokhlin's life was not easy. His father was executed in Stalin's Great Purge in 1941. Rokhlin himself was wounded while defending Moscow and spent a year or two in a German prisoner of war camp before managing to escape and eventually rejoin the Soviet army. After the war, he spent more than a year in a Soviet camp, since Stalin was very suspicious of returning war prisoners. Finally, after intercession by Kolmogorov and Pontryagin, he was released and served for a time as Pontryagin's assistant. In 1952, amid rumors that all ethnic Jews would be deported to the Far East, he was able to find a safer place in the north, at the Forestry Institute at Arkhangelsk, where he remained in a low level position for several years. Finally in 1959 he was able to obtain a real position at the State University in Leningrad, where his students included Yakov Eliashberg, Mikhail Gromov, Anatoly Vershik, and Oleg Viro.

A4.4. Freedman. In the case of an "even" intersection form, Freedman's theorem states more precisely that the Kirby-Siebenmann invariant can be identified with $\sigma / 8(\bmod 2)$, where $\sigma$ is the signature. On the other hand, in the "odd" case the Kirby-Siebenmann invariant and the intersection form can vary independently. Thus the "simplest" non-smoothable example has the homotopy type of the complex projective plane.

The Kirby-Siebenmann invariant is defined more generally for topological manifolds of any dimension as a cohomology class in $H^{4}(M ; \mathbb{Z} / 2)$. In dimensions strictly greater than four (or greater than five if $M$ has a boundary), this invariant vanishes if and only if the manifold has a triangulation which is locally piecewise linearly homeomorphic to Euclidean space. (In the case of a 4-manifold, we can say only that it vanishes if and only if $M \times \mathbb{R}$ has such a triangulation.) Similarly, given triangulations of two copies $M \times 0$ and $M \times 1$ as PL-manifolds, there is an obstruction in $H^{3}(M, \mathbb{Z} / 2)$ to extending to a triangulation of $M \times[0,1]$ as a PL-manifold.

Freedman's original proof was based on the concept of Casson handle (or "flexible handle"), a wild version of a thickened 2-dimensional disk. The figure in this section illustrates the related concept of a grope, which is due to CANNON [1978]. Compare the expositions of KIRBY [1989] or Scorpan 2005]. To me, Freedman's non-smoothable 4-manifolds are very mysterious objects. We know that they exist; but it doesn't seem to be possible to construct a completely explicit description for any one of them.

In recent years, Freedman has become an applied topologist. He is the Director of Microsoft's Station Q, which is attempting to use topological ideas to build a working quantum computer.

A4.5. Donaldson. Here is a very rough outline of Donaldson's argument. (Compare Donaldson [1983a, 1983b], DONALDSON AND KRONHEIMER 1990].) Given a Riemannian 4-manifold $M$ with positive definite intersection form, he chooses a specific $\mathrm{SU}_{2}$-bundle over $M$, and looks at the space of all "self-dual" connections (or "instantons") for this bundle, modulo the group of gauge automorphisms which map each fiber into itself. Using work of Cliff Taubes and others, he shows that this space is a smooth 5-dimensional manifold except for $n$ singular points, where $n$ is the number of pairs $\pm x \in H^{2}(M ; \mathbb{Z})$ such that $(x \cup x)[M]=1$. This 5-manifold can be compactified by adding a copy of $M$ at 
infinity. Furthermore, each singularity can be described as a cone over the complex projective plane $\mathbb{C P}^{2}$, suitably oriented. This yields a cobordism between $M$ and the disjoint union of $n$ copies of $\mathbb{C P}^{2}$. The existence of such a cobordism implies that the signature of $M$ is precisely equal to $n$; and it follows easily that the intersection form is diagonalizable.

A4.6. Taubes. See TAubes [1987], as well as Freedman 1984], GompF 1983, 1993], and DE Michelis AND FREEDMAN [1992]. I will not try to describe the Taubes construction, which is quite technical; but at least let me give a rough description of the first example of an exotic differentiable structure on $\mathbb{R}^{4}$.

Lemma 1. There exists a smooth closed 4-manifold $M$ and a topological splitting $M \cong M_{1} \# M_{2}$ as a connected sum, such that at least one of the two topological manifolds $M_{j}$ does not possess any differentiable structure.

Proof. Let $\mathbf{K} \subset \mathbb{C P}^{3}$ be the Kummer surface $x_{1}^{4}+x_{2}^{4}+x_{3}^{4}+x_{4}^{4}=0$. The intersection form of $\mathbf{K}$ is known to be of even type, with rank 22 and signature -16 . Let $-\mathbf{K}$ denote the same manifold with reversed orientation. Then it follows easily from Freedman's theorem that $-\mathbf{K}$ is oriented homeomorphic to the 5-fold topological connected sum $X \# X \# Y \# Y \# Y$, where $X$ is Freedman's $E_{8}$ manifold which has no differentiable structure, and where $Y=S^{2} \times S^{2}$. The conclusion follows.

Corollary. There exists a differentiable structure on the twice punctured 4-sphere with the following property: No smoothly embedded 3-sphere can separate the two puncture points 13

Proof. It follows from Lemma 1 that there exists a topological embedding of $S^{3} \times(0,1)$ into $M$ which separates the two summands $M_{1}$ and $M_{2}$. Now give this topologically embedded copy of $S^{3} \times(0,1)$ the differentiable structure which it inherits from $M$. If there were a smoothly embedded $S^{3}$ in this subset of $M$ which separates the two components of its topological boundary, then we could cut $M$ along this sphere and paste in two 4-disks in order to obtain two new smooth manifolds homeomorphic to $M_{1}$ and $M_{2}$ respectively. But by hypothesis, this is impossible. Since $S^{3} \times(0,1)$ is diffeomorphic to the twice punctured 4 -sphere, this completes the proof.

The next step is much harder. Let $M_{n}$ denote the connected sum of $X \# X$ with $n$ copies of $Y=S^{2} \times S^{2}$. Choose $n_{0}$ to be the smallest value such that $M_{n_{0}}$ has a smooth structure. (Thus $1 \leq n_{0} \leq 3$, using Donaldson's Theorem.)

Lemma 2 (Freedman). There is a compact subset $Q \subset S^{2} \times S^{2}$ with the following two properties:

- The complement $U=\left(S^{2} \times S^{2}\right) \backslash Q$ is homeomorphic to $\mathbb{R}^{4}$.

- Some neighborhood $V$ of $Q$, with $Q \subset V \subset\left(S^{2} \times S^{2}\right)$, can be embedded smoothly as a subset of $M_{n_{0}}$.

\footnotetext{
${ }^{13}$ For an early version of this result, see FREEDMAN 1979 .
} 
I will not try to describe the proof: Like the proof of Freedman's main theorem, it involves highly non-smooth constructions.

Assuming Lemma 2, the open set $U$, with the differentiable structure it inherits as a subset of $S^{2} \times S^{2}$, is the required exotic $\mathbb{R}^{4}$. In fact if $U$ were diffeomorphic to $\mathbb{R}^{4}$, then it would be the union of an increasing sequence $B_{1} \subset B_{2} \subset \cdots$ of smoothly embedded copies of the closed unit 4-ball. For large $j$, the boundary sphere $\partial B_{j}$ would be contained in $V$, and hence would map to a smoothly embedded sphere in $M_{n_{0}}$, which would split $M_{n_{0}}$ as an impossible smooth connected sum.

There is a large literature about differentiable structures and diffeomorphism invariants of 4-manifolds. See for example [FRIEDMAN AND MORGAN [1989], SAlAmon 1999], and Morgan [2003].

A4.7. What Next? It was a big surprise in the 1960s to discover that dimensions 3 and 4 are the most difficult cases - higher dimensions are much easier. For some explanation of why this is true, see FREEDMAN [1984]. A quick survey of the higher dimensional theory is given in MILNOR 2011]; in particular, Tables 2 and 3 of that paper describe the precise structure of the group $\mathscr{S}_{n}$ for every $n \neq 4$ between one and sixty-three.

\section{ACKNOWLEDGMENTs}

This paper was written with substantial help from Araceli Bonifant, Lucienne Pereira, Ragni Piene, Andrew Ranicki, and Scott Sutherland. I also want to thank Thomas Costa, Michael Freedman, Susan Friedlander, Etienne Ghys, Claude LeBrun, Misha Lyubich, Marius Thaule, Oleg Viro, the Swiss Mathematical Society, and the St. Petersburg Mathematical Society for their help. Finally, I want to thank the Norwegian Academy of Science and Letters for its support, and the Organizing Committee of the Seoul ICM for an inspiring meeting.

\section{PiCTURE CREDITS}

\section{Portraits:}

Niels Henrik Abel: photograph of the memorial in Gjerstad, Norway, (C)Torgrim Landsverk, Norwegian Academy of Science and Letters; public domain.

James Alexander and wife: photographer unknown; probably taken in the French Alps. Photograph courtesy of the Alexander Family; from the Shelby White and Leon Levy Archives Center, Institute for Advanced Study, Princeton, NJ, USA.

Augustin Louis Cauchy: portrait by Charles H. Reutlinger; public domain.

Walther (von) Dyck: sketch by Lucienne Pereira, used by permission of the artist. 
Leonhard Euler: the Soviet stamp in $§ 1.1$ commemorates Euler's 250th birthday. The portrait in $\S 1.2$ is by Jakob Emanuel Handmann; public domain.

Carl Friedric Gauss: portrait by Christian Albrecht Jensen; public domain.

Wolfgang Haken: from the Paul R. Halmos Photograph Collection, e_ph_0119_01, The Dolph Briscoe Center for American History, The University of Texas at Austin.

Richard Hamilton: sketch by Lucienne Pereira, used by permission of the artist.

Poul Heegaard: used by permission from The Norwegian Academy of Science and Letters.

Hellmuth Kneser: used by permission of the Archives of the Mathematisches Forschungsinstitut Oberwolfach.

Paul Koebe: sketch by Lucienne Pereira, used by permission of the artist.

Simon L'Huilier: this is an 1835 painting by Joseph Hornung, owned by the Société des Arts in Geneva; public domain.

A. A. Markov, Jr.: used by permission of Margarita Markova, with many thanks for allowing us to use this picture of A. A. Markov, Jr., from his family collection in this publication.

August Ferdinand Möbius: portrait by Adolf Neumann; public domain.

Edwin Moise: from the Paul R. Halmos Photograph Collection, e_ph_0208_01, The Dolph Briscoe Center for American History, The University of Texas at Austin.

George D. Mostow: used by permission of Michael Marsland/Yale University.

Christos Papakyriakopoulos: sketch by Lucienne Pereira, used by permission of the artist.

Grigori Perelman: photograph by George M. Bergman, used by permission of the Archives of the Mathematisches Forschungsinstitut Oberwolfach.

Henri Poincaré: in $\S 2.6$ and $\S 3.2$; public domain.

Tibor Radó: sketch by Lucienne Pereira, used by permission of the artist.

Bernhard Riemann: public domain.

Vladimir Rokhlin: used by permission of the Archives of the Mathematisches Forschungsinstitut Oberwolfach.

Herbert Seifert: used by permission of the Archives of the Mathematisches Forschungsinstitut Oberwolfach.

William Thurston: the photograph in $\S 3.10$ is used by permission of Thomas Sharland. The photograph in $\S 3.12$ is taken from a video of his talk at the conference "Frontiers in Complex Dynamics" in Banff, Canada, 2011. Used by permission of Scott Sutherland.

Friedhelm Waldhausen: used by permission of Andrew Ranicki.

Hermann Weyl: ETH-Bibliothek Zürich, Bildarchiv; public domain. 
J. H. C. Whitehead: used by permission of Oliver Whitehead.

The pictures of: Simon Donaldson, Michael Freedman, William Jaco, Klaus Johannson, Peter Shalen, and Clifford Taubes are used by permission of the respective subjects.

\section{Illustrations:}

Diagrams by John Milnor: graph for the bridge problem in $\S 1.1$; surface of a polyhedron in Euclidean 3-space in $\S 2.1$; picture of genus 2 surface and polygon in $\S 2.3$; pictures of $E_{1}, E_{2}, E_{3}$ in $\S 2.4$ as well as figure at bottom of p. 552 , picture to the right of Kneser's picture in $§ 3.4$.

Diagrams by Scott Sutherland: winding number figure in $\S 1.3$; link figure in $\S 1.4$; pictures of simply connected, double connected, triply connected, and another triply connected surface in $\S 2.3$; torus in $\S 2.4$; picture of sphere $E_{0}$ in $\S 2.4$; pictures of sphere, tours and 2-torus in $\S 2.7$. Each is used by permission.

Complicated surface in 3-space: scan from the paper MöBIUS [1863]; see p. 552 .

Dodecahedron: public domain; see p. 546.

Figure-eight knot: By Jim.belk; public domain; see p. 561

Königsberg in the 18th century: by Bogdan Giuşcă; public domain; see p. 545 .

Sphere with 3-handles: by Oleg Alexandrov using MATLAB; public domain; see p. 553.

Picture of horned sphere: in $§ 3.3$. Taken from Topology by Hocking and Young, Addison-Wesley 1961; Dover 1988, p. 176. Used by permission of Dover Publications, Inc.

The grope: in $\S 4.4$. Taken from CANNON [1978], used by permission of the American Mathematical Society; see p. 568 .

Whitehead link: public domain; see p. 562

\section{REFERENCES}

S. I. Adyan [1955], Algorithmic unsolvability of problems of recognition of certain properties of groups (Russian), Dokl. Akad. Nauk SSSR (N.S.) 103, 533-535. MR0081851 (18,455e)

J. W. Alexander [1915], A proof of the invariance of certain constants of Analysis situs, Trans. Amer. Math. Soc. 16, no. 2, 148-154. MR1501007

J. W. Alexander [1922], A proof and extension of the Jordan-Brouwer separation theorem, Trans. Amer. Math. Soc. 23, no. 4, 333-349. MR1501206

J. W. Alexander [1924], On the subdivision of 3-space by a polyhedron, Proc. Nat. Acad. Sci. 10, $6-8$.

J. W. Alexander [1928], Topological invariants of knots and links, Trans. Amer. Math. Soc. 30, no. 2, 275-306. MR.1501429

P. Alexandroff and H. Hopf [1935], Topologie, Springer, Berlin.

L. Bieberbach [1911], Über die Bewegungsgruppen der Euklidischen Räume I, Math. Ann. 70, no. 3, 297-336. MR151123 
L. Bieberbach [1912], Über die Bewegungsgruppen der Euklidischen Räume (Zweite Abhandlung.) Die Gruppen mit einem endlichen Fundamentalbereich, Math. Ann. 72, no. 3, 400-412. $\operatorname{MR} 1511704$

A. Borel [1981], Commensurability classes and volumes of hyperbolic 3-manifolds, Ann. Scuola Norm. Sup. Pisa, Cl. Sci. (4) 8, no. 1, 1-33. MR616899 (89j:22008)

J. Cannon [1978], The recognition problem: What is a topological manifold? Bull. Amer. Math. Soc. 84, no. 5, 832-866. MR0494113(58 \#13043)

A. Casson [1986], Three lectures on new-infinite constructions in 4-dimensional manifolds, Á la recherche de la topologie perdue, Progr. Math., vol. 62, Birkhäuser Boston, Boston, pp. 201244. MR.900253

A. Clebsch [1865], Ueber diejenigen ebenen Curven, deren Coordinaten rationale Functionen eines Parameters sind, J. reine ang. Math. 64, 43-65.

http://www.maths.ed.ac.uk/ aar/papers/clebschgenus.pdf

R. H. Crowell and R. H. Fox [1963], Introduction to knot theory (Reprint of the 1963 original), Graduate Texts in Mathematics, no. 57, Springer-Verlag, New York-Heidelberg. MR0445489 (56 \#3829)

S. De Michelis and M. Freedman [1992], Uncountably many exotic $\mathbb{R}^{4}$ 's in standard 4-space, J. Diff. Geom. 35, no. 1, 219-254. MR1152230 (93d:57036)

J. Dieudonné [1988], A history of algebraic and differential topology. 1900-1960, Birkhäuser Boston, Boston. MR.995842 (90g:01029)

S. Donaldson [1983a], Self-dual connections and the topology of smooth 4-manifolds, Bull. Amer. Math. Soc. 8, no. 1, 81-83. MR682827 (84e:57016)

S. Donaldson [1983b], An application of gauge theory to four-dimensional topology, J. Diff. Geom. 18, no. 2, 279-315. MR710056 (85c:570015)

S. Donaldson and P. Kronheimer [1990], The geometry of four-manifolds, Oxford Mathematical Monographs, Oxford Science Publications, The Clarendon Press, Oxford University Press, New York. MR1079726 (92a:57036)

W. Dyck [1888], Beiträge zur Analysis situs, Ein- und zweidimensionale Mannigfaltigkeiten (German), Math. Ann. 32, no. 4, 457-512. MR1510522 https://eudml.org/doc/157390

F. Enriques [1905], Sulla proprietà caratteristica delle superficie algebriche irregolari, Rend. Accad. Sci. Bologna, 9, 5-13.

M. H. Freedman [1979], A fake $S^{3} \times \mathbb{R}$, Ann. of Math. (2) 110, no. 1, 177-201. MR541336 (80j:57029)

M. H. Freedman [1982], The topology of four-dimensional manifolds, J. Diff. Geom. 17, no. 3, 357-453. MR679066 (84b:57006)

M. H. Freedman [1984], There is no room to spare in four-dimensional space, Notices Amer. Math. Soc. 31, no. 1, 3-6. MR728340 (85k:57012)

M. Freedman and F. Quinn [1990], The topology of 4-manifolds, Princeton Mathematical Series, vol. 39, Princeton University Press, Princeton, NJ. MR.1201584 (94b:57021)

M. Freedman, R. Gompf, S. Morrison and K. Walker [2010], Man and machine thinking about the smooth 4-dimensional Poincaré conjecture, Quantum Topol. 1, no 2, 171-208. MR2657647 (2011f:57061)

A. Friedman [1922], Über die Krümmung des Raumes, Z. Physik 10, 377-386.

R. Friedman and J. Morgan [1989], Complex versus differentiable classification of algebraic surfaces, Proceedings of the 1987 Georgia Topology Conference (Athens, GA, 1987). Topology Appl. 32, no. 2, 135-139. MR1007985 (90f:57039) 
C. F. Gauss [1833], Zur mathematischen Theorie der electrodynamischen Wirkung, Königliche Gesellschaft der Wissenschaften zu Göttingen 5, 602-629.

H. Gieseking [1912], Analytische Untersuchungen über topologische Gruppen, Thesis, Muenster.

R. Gompf [1983], Three exotic $\mathbf{R}^{4}$ 's and other anomalies, J. Diff. Geom. 18, no. 2, 317-328. MR710057 (85b:57038)

R. Gompf [1993], An exotic menagerie, J. Diff. Geom. 37, no. 1, 199-223. MR1198606|(93k:57041)

C. Gordon and J. Luecke [1989], Knots are determined by their complements, J. Amer. Math. Soc. 2, no. 2, 371-415. MR965210(90a:57006a)

J. Gray [2013], Henri Poincaré, a scientific biography, Princeton University Press, Princeton, NJ. (See p. 230.) MR2986502

M. Gromov [1979-1980], Hyperbolic manifolds (according to Thurston and Jørgensen), Séminaire Bourbaki, Vol. 1979/80, Lecture Notes in Mathematics, vol. 842, Springer, Berlin-New York, pp. 40-53 (available through Numdam). MR636516 (84b:53046)

W. Haken [1962], Über das Homöomorphieproblem der 3-Mannigfaltigkeiten. I, Math. Z. 80, 89-120. MR0160196 (28 \#3410)

R. Hamilton [1982], Three-manifolds with positive Ricci curvature, J. Diff. Geom. 17, no. 2, 255-306. MR664497 (84a:53050)

P. Heegaard [1898], Forstudier til en topologisk teori for algebraiske Sammenhäng, Copenhagen, det Nordiske Forlag Ernst Bojesen.

http://www.maths.ed.ac.uk/ aar/papers/heegaardthesis.pdf http://www.maths.ed.ac.uk/ aar/papers/heegaardenglish.pdf

J. Hempel [1976], 3-manifolds, Ann. of Math. Studies, No. 86, Princeton University Press, Princeton, NJ; University of Tokyo, Tokyo. MR0415619 (54 \#3702)

F. Hirzebruch [1966], Topological methods in algebraic geometry, Classics in Mathematics, Springer-Verlag, Berlin.

F. Hirzebruch [1999], Emmy Noether and topology: the heritage of Emmy Noether (Ramat-Gan, 1996), 57-65, Israel Math. Conf. Proc., 12, Bar-Ilan Univ., Ramat Gan, 1999. MR 1665435 (2000c:01032)

F. Hirzebruch and M. Kreck [2009], On the concept of genus in topology and complex analysis, Notices Amer. Math. Soc., 56, no. 6, 713-719. MR2536790(2010h:57030)

H. Hopf [1925], Zum Clifford-Kleinschen Raumproblem, Math. Ann. 95, no. 1, 313-319.

W. Jaco and P. Shalen [1978], "A new decomposition theorem for irreducible sufficiently-large 3-manifolds", Algebraic and geometric topology (Proc. Sympos. Pure Math., Stanford University, Stanford, CA, 1976), Proc. Sympos. Pure Math., XXXII, Amererican Mathematical Society, Providence, R.I., pp. 71-84. MR520524 (80j:57008)

I. M. James (editor) [1999], History of Topology, North-Holland, Amsterdam. MR.1674906 (2000g:00032)

K. Johannson [1979], Homotopy equivalences of 3-manifolds with boundaries, Lecture Notes in Mathematics, vol. 761, Springer, Berlin. MR551744 (82c:57005)

T, Jørgensen [1977], Compact 3-manifolds of constant negative curvature fibering over the circle, Ann. of Math. (2) 106, no. 1, 61-72. MR0450546 (56 \#8840)

M. Kervaire and J. Milnor [1960], Bernoulli numbers, homotopy groups, and a theorem of Rohlin, Proc. Internat. Congress Math., 1958, pp. 454-458, Cambridge University Press, New York. MR0121801 (22 \#12531) Also in Collected Papers of John Milnor: III. Differential topology, American Mathematical Society, Providence, RI, 2007, pp. 243-247. MR2307957(2008h:01022)

M. Kervaire and J. Milnor [1963], Groups of homotopy spheres. I, Ann. of Math. (2) 77, 504-537. MR0148075 (26 \#5584) Also in Collected Papers of John Milnor: III. Differential topology, American Mathematical Society, Providence, RI, 2007, pp. 89-122. MR2307957(2008h:01022) 
R. Kirby [1989], The topology of 4-manifolds, Lecture Notes in Mathematics, vol. 1374, SpringerVerlag, Berlin. MR1001966 (90j:57012)

R. Kirby and L. Siebenmann [1969], On the triangulation of manifolds and the Hauptvermutung, Bull. Amer. Math. Soc. 75, 742-749. MR0242166 (39 \#3500)

R. Kirby and L. Siebenmann [1977], Foundational essays on topological manifolds, smoothings, and triangulations. With notes by John Milnor and Michael Atiyah, Annals of Mathematics Studies, No. 88, Princeton University Press, Princeton, NJ. MR0645390 (58 \#31082)

O. A. Laudal and R. Piene (editors) [2002], The legacy of Niels Henrik Abel, Springer-Verlag, Berlin. MR2074480 (2005a:00019)

S. Lefschetz [1924], L'Analysis situs et la géometrie algébrique, Gauthier-Villars, Paris. $\operatorname{MR} 0033557(11,456 \mathrm{c})$

S. Lhuilier and J. Gergonne [1812-1813], Mémoire sur la polyédrométrie $\cdots$, Ann. Math. Pures et Appl. (= Ann. de Gergonne) 3, 169-189. http://www.numdam.org/item?id=AMPA_1812-1813__3__169_0

W. B. R. Lickorish [1997], An introduction to knot theory, Graduate Texts in Mathematics, vol. 175, Springer-Verlag, New York. MR1472978 (98f:57015)

J. B. Listing [1862], Der Census räumlicher Complexe oder Verallgemeinerung des Euler'schen Satzes von den Polyedern, Abhandlungen königlichen Gesellschaft der Wissenschaften zu Göttingen 10, 97-180. http://www.maths.ed.ac.uk/ aar/papers/listing2.pdf

C. Manolescu [2014], An introduction to knot Floer homology, ArXiv:1401.7107.

A. A. Markov, Jr. [1958], The insolubility of the problem of homeomorphy (Russian), Dokl. Akad. Nauk SSSR 121, 218-220. MR0097793 (20 \#4260)

J. Milnor [1958], "On simply connected 4-manifolds", in Symposium Internacional de Topología Algebraica (International Symposium on Algebraic Topology), Universidad Nacional Autónoma de México and UNESCO, Mexico City, pp. 122-128. MR.0103472(21 \#2240) Also in Collected Papers of John Milnor: IV. Homotopy, homology and manifolds, American Mathematical Society, Providence, RI, 2009, pp. 151-157. MR2590677 (2011g:01020)

J. Milnor [1962], A unique decomposition theorem for 3-manifolds. Amer. J. Math., 84, 1-7. MR0142125(25 \#5518) Also in Collected Papers of John Milnor: II. The fundamental group, American Mathematical Society, Providence, RI, 1995, pp. 237-243. MR2569311

J. Milnor [1987], The work of M. H. Freedman, Proc. Internat. Congr. Math., Berkeley, CA, 1986, pp. 13-15. MR934211 (89d:01064) Also in Collected Papers of John Milnor: IV. Homotopy, homology and manifolds, American Mathematical Society, Providence, RI, 2009, pp. 321-323. MR 2590677 (2011g:01020)

J. Milnor [2011], Differential topology forty-six years later, Notices Amer. Math. Soc., 58, no. 6, 804-809. MR2839925(2012i:57001)

J. Milnor and D. Husemoller [1973], Symmetric bilinear forms. Ergebnisse der Mathematik und ihrer Grenzgebiete, Band 73. Springer-Verlag, New York-Heidelberg. MR0506372(58 \#22129)

A. F. Möbius [1863], Theorie der elementaren Verwandtschaft, Berichte Königlich Sächsischen Gesellschaft der Wissenschaften zu Leipzig 15, 18-57. http://www.maths.ed.ac.uk/ aar/papers/mobiussurf.pdf

E. Moise [1952], Affine structures in 3-manifolds. V. The triangulation theorem and Hauptvermutung, Ann. of Math. (2) 56, 96-114. MR0048805 (14,72d)

J. Morgan [2003], "Definition of the Seiberg-Witten (SW) invariants of 4-manifolds", Low dimensional topology, New Stud. Adv. Math., vol. 3, International Press, Somerville, MA, pp. 1-11. MR 2052242 
J. Morgan and G. Tian [2007], Ricci flow and the Poincaré conjecture, Clay Mathematics Monographs, vol. 3, American Mathematical Society, Providence, RI; Clay Mathematics Institute, Cambridge, MA. MR.2334563 (2008d:57020)

J. Morgan and G. Tian [2014], The geometrization conjecture, Clay Mathematics Monographs, vol. 5, American Mathematical Society, Providence, RI; Clay Mathematics Institute, Cambridge, MA. MR 3186136

G. D. Mostow [1968], Quasi-conformal mappings in n-space and the rigidity of the hyperbolic space forms, Inst. Hautes Études Sci. Publ. Math., 34, 53-104. MR.0236383 (38 \#4679)

W. Neumann and D. Zagier [1985], Volumes of hyperbolic 3-manifolds, Topology 24, no. 3, 307-332. MR815482(87j:57008)

C. D. Papakyriakopoulos [1957], On Dehn's lemma and the asphericity of knots, Ann. of Math. (2) 66, 1-26. MR0090053(19,761a)

E. Picard and G. Simart $[1897,1906]$, Théorie des fonctions algébriques de deux variables indépendantes $I$ and II, Gauthier-Villars, Paris.

H. Poincaré [1900], Second complément à l'analysis situs, Proc. London Math. Soc. 32, no. 1, 277-308 (Oeuvres 6, 338-370).

H. Poincaré [1902], Quatriéme complément à l'analysis situs, Journ. de Math. 8, 169-214 (Oeuvres 6, 397-434).

H. Poincaré [1904], Cinquième complément à l'analysis situs, Rend. Palermo 18, 45-110 (Oeuvres 6, 435-498).

Also see Papers on topology: Analysis situs and its five supplements, translated and introduced by J. Stillwell, History of Mathematics, vol. 37, American Mathematical Society, Providence, RI, and London Mathematical Society, London, 2010. MR2723194 (2011j:01017)

J. C. Pont [1974], Petite enfance de la topologie algébrique (French), Enseignement Math. (2) 20 111-126. MR0373806 (51 \#10006)

G. Prasad [1973], Strong rigidity of Q-rank 1 lattices, Invent. Math. 21, 255-286. MR0385005 (52 \#5875)

T. Radó [1924], Über den Begriff der Riemannschen Fläche, Acta Sc. Math. Szeged 2, 101-121.

B. Riemann [1857], Theorie der Abel'schen Functionen, J. reine ang. Math 54. (See pages 89 and 97 of the following.) https://ia700400.us.archive.org/16/items/bernardrgesamm00riemric/ bernardrgesamm00riemrich.pdf

R. Riley [2013], A personal account of the discovery of hyperbolic structures on some knot complements, Expo. Math. 31, no. 2, 104-115. MR3057120

V. Rokhlin [1952], New results in the theory of four-dimensional manifolds (Russian), Dokl. Akad. Nauk SSSR (N.S.) 84, 221-224. MR0052191(14,573b)

V. Rokhlin [1958], Relations between characteristic classes of four-dimensional manifolds (Russian), Kolomen. Ped. Inst. Uč. Zap. Ser. Fiz.-Mat. 2, no. 1, 3-17. MR0133138(24 \#A2972)

D. Rolfsen [1976], Knots and links, Mathematics Lecture Series, No. 7, Publish or Perish, Inc., Berkeley, CA. MR0515288(58 \#24236)

D. Salamon [1999], Spin geometry and Seberg-Witten invariants, at http://www.math.ethz.ch/ salamon/PREPRINTS/witsei.pdf

A. Scorpan [2005], The wild world of 4-manifolds, American Mathematical Society, Providence, RI. MR2136212(2006h:57018)

H. Seifert and W. Threlfall [1934], Lehrbuch der Topologie, Teubner, Leipzig. (English translation, Academic Press 1980).

J.-P. Serre [1970], Cours d'arithmétique (French), Collection SUP: "Le Mathématicien", vol. 2, Presses Universitaires de France, Paris. MR0255476 (41 \#138) 
S. Smale [1961], Generalized Poincaré's conjecture in dimensions greater than four, Ann. of Math. (2) 74, 391-406. MR0137124 (25 \#580)

J. Stallings [1962], The piecewise-linear structure of Euclidean space, Proc. Cambridge Philos. Soc. 58, 481-488. MR0149457 (26 \#6945)

C. Taubes [1987], Gauge theory on asymptotically periodic 4-manifolds, J. Diff. Geom. 25, no. 3, 363-430. MR882829 (88g:58176)

W. Thurston [1980], The geometry and topology of three-manifolds, Princeton University Lecture Notes, available at http://www.msri.org/publications/books/gt3m

W. Thurston [1982], Three-dimensional manifolds, Kleinian groups and hyperbolic geometry, Bull. Amer. Math. Soc. 6, no. 3, 357-381. MR648524 (83h:57019)

W. Thurston [1997], Three-dimensional geometry and topology. Vol. 1 (Edited by Silvio Levy), Princeton Mathematical Series, vol. 35, Princeton University Press, Princeton, NJ. MR.1435975 (97m:57016)

F. Waldhausen [1968], On irreducible 3-manifolds which are sufficiently large, Ann. of Math. (2) 87, 56-88. MR0224099 (36 \#7146)

J. H. C. Whitehead [1949a], On simply connected, 4-dimensional polyhedra, Comment. Math. Helv. 22, 48-92. MR0029171 (10,559d)

J. H. C. Whitehead [1949b], Combinatorial homotopy. I, Bull. Amer. Math. Soc. 55, 213-245. MR0030759(11,48b)

D. Zagier [1986], Hyperbolic manifolds and special values of Dedekind zeta-functions. Invent. Math. 83, no. 2, 285-301. MR818354 (87e:11069)

Institute for Mathematical Sciences, Stony Brook University, New York

E-mail address: jack@math.sunysb.edu 